\title{
Light Meson Mass Spectra with Massive Quarks
}

\author{
Teruo Kurai \\ IEEE Member, 1-32-1 Nishifu-Machi, Fuchu-Shi, Tokyo, Japan \\ Email: kurai.teruo@topaz.plala.or.jp
}

How to cite this paper: Kurai, T. (2021) Light Meson Mass Spectra with Massive Quarks. Journal of Modern Physics, 12, 1545-1572.

https://doi.org/10.4236/jmp.2021.1211093

Received: August 12, 2021

Accepted: September 13, 2021

Published: September 16, 2021

Copyright $\odot 2021$ by author(s) and Scientific Research Publishing Inc. This work is licensed under the Creative Commons Attribution International License (CC BY 4.0).

http://creativecommons.org/licenses/by/4.0/

\begin{abstract}
We investigate light meson mass spectra with massive $\mathrm{u}, \mathrm{d}$, and $\mathrm{s}$ quarks and with a spin effect under a bound system in $3+1$ dimensional QCD by using the first order perturbation correction. In the process of determining charged kaon and neutral kaonmasses, we obtain masses of $\mathrm{u}, \mathrm{d}$, and $\mathrm{s}$ quarks that are slightly smaller than the currently accepted values. Using these masses, we obtain light meson mass spectra that includes mass splitting of charged and neutral kaons and $\rho$ mesons. The most interesting of our results is that the pion mass remains unchanged even though $\mathrm{u}, \mathrm{d}$, and $\mathrm{s}$ quarks become massive.
\end{abstract}

\section{Keywords}

Light Meson Mass, Quark Mass, Pion Mass, Quantum Chromodynamics

\section{Introduction}

It is well understood that properly explaining meson properties such as mass spectra, decay constants, and the pion form factor (the pion wave function in momentum space) is fundamental to understanding hadron physics because a meson is the simplest composite particle system. It is especially important to explain meson properties within a quantum chromodynamics (QCD) framework because it is widely accepted that the interactions between quarks (or antiquarks) are described by QCD. Currently, there are two main approaches to explaining meson properties. One is based on the consideration that covariance should be the first priority when describing mesons. The other approach of describing mesons without setting the covariance as the first priority was developed mainly to investigate mass spectra. The mass spectra and pion wave functions in momentum space (pion form factor) resulting from these two descriptions are different. Although it is well-known that the former description cannot explain the 
$\frac{1}{2}$ property of pion mass spectra, the latter description can explain light meson mass spectra (including pion mass spectra) fairy well, as shown by Braw and Semay [1], Choi et al. [2] and our published results [3]. Significantly, the main terms of the corresponding eigenfunction are Gaussian in both the configuration space ( $\mathrm{r}$ space) and momentum space in all three references even though the approaches are different. In addition, the two descriptions give different results in the case of the t'Hooft problem, in which mass spectra in two dimension can be dealt with exactly. The former description cannot obtain a zero mass simultaneously with a nonzero mass spectrum, as shown by t'Hooft [4] and Hornbostwl et al. [5]. By contrast, the author [6] showed the existence of zeromass simultaneously with a nonzero mass spectrum, which is consistent with the results of t'Hooft. For a pion wave function in momentum space, it is also well known that the former description gives Reggae-like profile functions. For example, inMello et al. [7], a pion is described by three simple pole solutions in $r$ space (Reggae-like functions in momentum space),whereas in the latter case, the description for satisfying light meson mass spectra (including a pion mass spectrum) is Gaussian in momentum space, as mentioned before. In addition, for considering the pion electromagnetic form factor, the following three methods are proposed. The first is the covariant spectator theory (CST) by Biernat et al., but this wave function is Reggae-like [8]. The second is Dyson-Schwinger equation method, but this wave function is not Gaussian either, as shown in Chang et al. [9]. In addition, according to Arrington et al. [10], the pion valence quark distribution function (DF), $q^{\pi}(x, \zeta)$, describes behavior of hadron wave functions at large valence-quark relative momenta. Numerous analyses predict the following large- $x$ behavior $q^{\pi}\left(x, \zeta=\zeta_{H}\right) \sim(1-x)^{\beta}$. Here $x$ is a light-front fraction of the system's total momentum at resolving scale $\zeta$. The apparent $\beta$ exponent can range between $\sim 1$ and $\sim 2.5$. The Drell-Yan-West relation provides a link between the large behavior of DFs and the large $-Q^{2}$ dependence of hadron elastic form factors of which leading elastic electromagnetic form factor scales as $\left(1 / Q^{2}\right)^{n}$. For a pseudo-scalar meson, $n=2$ and $q^{\pi}\left(x, \zeta_{H}\right) \sim(1-x)^{2}$. Thus, this DFs derive Reggae-like wave functions. The third is the Drell-Yan frame shown by Li et al. [11]. The Drell-Yan frame restores dynamical covariance but does not include zero-mode contribution which needs to fit to the pion electromagnetic form factor. To include zero-mode contribution lose the dynamical covariance. The most recent experimental results by the Jefferson Lab Hall A collaboration [12] show that the t-dependence of the cross section, usually parametrized by Reggae-like profile functions, is no longer valid at typical values of $-t>1 \mathrm{Gev}^{2}$, and that a fitting form of $t$ dependence is $\exp \left(-B t^{\prime}\right)$ where $t^{\prime}=t_{\min }-t$, $t=\left(q-q^{\prime}\right)^{2}, q$ and $q^{\prime}$ are photon and $\pi^{0}$ momentum, respectively. This shows that $\pi^{0}$ is described as Gaussian in momentum space. To consider this experimental result seriously, Reggae like profile functions for pion wave function should be reconsidered. For decay constants, both the former and the latter 
descriptions give satisfactory results, as shown in Aoki et al. [13] for the former (although the wave function is not Gaussian) and in Ref [2] for the latter (a Gaussian description). Based on these comparisons, the Gaussian description seems to be better for mesons, or at least for light mesons. However, the Gaussian description has not been given a global physics rule like covariance for the former description. This problem should be considered seriously. Fitting of the light meson mass spectra in Ref. [3] is not good enough. Instead, these results are taken for granted because these are obtained under the chiral limit condition, which sets the masses of $\mathrm{u}, \mathrm{d}$, and s quarks at zero without considering the spin effect for vector mesons. In this paper, we investigate light meson mass spectra under the conditions that $\mathrm{u}, \mathrm{d}$, and $\mathrm{s}$ quarks are massive and that vector mesons have a spin effect.

\section{Formulation}

We previously showed the chiral limit of light meson mass spectra [3]. Here we extend our method to the non-chiral limit case in which the masses of $\mathrm{u}, \mathrm{d}$, and $\mathrm{s}$ quarks are non-zero and there is a spin effect for vector mesons. To do this, we recall the Dirac equation in QCD with a mass term. The Dirac equation is expressed as

$$
i \frac{\partial q}{\partial t}=-i \alpha^{k} D_{A k} q-\beta m q
$$

The Dirac equation of the complex conjugate $q^{\dagger}$ becomes as the following.

$$
i \frac{\partial q^{\dagger}}{\partial t}=-i \alpha^{k} D_{A k} q^{\dagger}+\beta m q^{\dagger}
$$

where $D_{A k} \equiv \partial_{k}-i g A_{k}^{a}\left(\frac{\lambda_{a}}{2}\right)$.

The $\frac{\lambda_{a}}{2}$ components are generators of the adjoint representation of the color gauge group.

We employ the metric system and $\gamma$ matrices as follows, according to Weinberg [14].

$$
\begin{gathered}
\eta^{00}=-1, \eta^{11}=\eta^{22}=\eta^{33}=1 \\
\gamma^{0}=(-i)\left(\begin{array}{cc}
0 & \sigma_{0} \\
\sigma_{0} & 0
\end{array}\right), \gamma^{k}=(-i)\left(\begin{array}{cc}
0 & \sigma_{k} \\
-\sigma_{k} & 0
\end{array}\right)
\end{gathered}
$$

where $\sigma_{0}$ is a unit matrix of a $2 \times 2$ matrix and $\sigma_{k}$ is the $2 \times 2$ Pauli-matrix specified by $(k=1,2,3)$

$$
\alpha^{k}=\gamma^{0} \gamma^{k} \text { and } \beta=i \gamma^{0}
$$

First, we briefly describe our formalism and the equation of motion we obtained previously [3]. Suura [15] [16] defined the Bethe-Salpeter-like amplitude as

$$
\chi(1,2)=\langle 0|q(1,2)| P\rangle
$$


where $|0\rangle$ and $|P\rangle$ denote the vacuum and physical states, respectively, and the gauge invariant bi-local operator $q(1,2)$ is defined in the non-Abelian gauge field as

$$
q(1,2) \equiv T_{r}^{c} q_{\eta}^{\dagger}(2) P \exp \left(i g \int_{1}^{2} \mathrm{~d} \vec{x} \vec{A}^{a}(\vec{x})\left(\frac{\lambda_{a}}{2}\right)\right) q_{\xi}(1)
$$

Here $\xi$ and $\eta$ denote the Dirac indices, $P$ denotes the path ordering, and the $\frac{\lambda_{a}}{2}$ components are generators of the adjoint representation of the $\mathrm{SU}(\mathrm{N})$ color gauge group, as mentioned previously. The trace is calculated for color spin a. The Dirac equation for quarks and antiquarks shows the dependence of local gauge fields. Thus, the operator $q(1,2)$ would be gauge variant if the string term is absent. However, because of the existence of the string term, the defined operator of Equation (3) is not dependent of gauge fields explicitly, as shown in Ref. [17]. In this sense, the defined operator is gauge invariant but path dependent. Because the physical properties of an observable color singlet should be the path independent, Suura chose a straight line for the zeroth order [15]. We also adopt this choice to investigate chiral limit light meson mass splitting [3]. For the chiral limit case, the starting equation of motion is the following as given in Ref. [3].

$$
\begin{aligned}
i \frac{\partial}{\partial t} q(1,2)= & -i \vec{\alpha} \cdot \vec{\nabla}(2) q(1,2)-q(1,2) i \vec{\alpha} \cdot \vec{\nabla}(1) \\
& +g \int_{1}^{2} \mathrm{~d} \vec{x} q_{E}(1,2 ; x)+g \vec{\alpha} \cdot \int_{1}^{2} \mathrm{~d} \vec{x} \times q_{B}(1,2 ; x)
\end{aligned}
$$

Thus, for the non-chiral limit case, from Equation (1) and Equation (2) leads to the starting equation of motion as follows.

$$
\begin{aligned}
i \frac{\partial}{\partial t} q(1,2)= & -i \vec{\alpha} \cdot \vec{\nabla}(2) q(1,2)-q(1,2) i \vec{\alpha} \cdot \vec{\nabla}(1)+\beta m_{2} q(1,2)-q(1,2) \beta m_{1} \\
& +g \int_{1}^{2} \mathrm{~d} \vec{x} q_{E}(1,2 ; x)+g \vec{\alpha} \cdot \int_{1}^{2} \mathrm{~d} \vec{x} \times q_{B}(1,2 ; x)
\end{aligned}
$$

where $q_{\bar{O}}(1,2 ; x) \equiv q^{\dagger}(2) U(2, x) \overline{O^{a}}\left(\frac{\lambda_{a}}{2}\right) U(x, 1) q(1) \quad O$ is any operator

$$
U(1,2) \equiv P \exp \left(i g \int_{1}^{2} \mathrm{~d} \vec{x} \vec{A}^{a}(\vec{x})\left(\frac{\lambda_{a}}{2}\right)\right)
$$

Here we adopt the center of mass of the system and relative coordinates as

$$
\begin{gathered}
\vec{G}=\frac{m_{1} \vec{r}(1)+m_{2} \vec{r}(2)}{m_{1}+m_{2}} \\
\vec{r}=\vec{r}(2)-\vec{r}(1)
\end{gathered}
$$

where $\vec{r}(2), \vec{r}(1)$ denote the point 2 and 1 , respectively.

In the relative coordinates and in the rest frame, we obtain the kinetic term as follows.

$$
-i \vec{\alpha} \cdot \vec{\nabla}(2) q(1,2)-q(1,2) i \vec{\alpha} \cdot \vec{\nabla}(1)=-[i \vec{\alpha} \cdot \vec{\nabla}(\vec{r}), q(t ; \vec{r})]
$$


This is same as in the chiral limit case [3].

Because quarks and antiquarks are point-like particles and our string is considered to be a straight line, the three dimensional integral for the string can be written as

$$
\int_{1}^{2} \mathrm{~d} \vec{x}=\int_{z_{1}}^{z_{2}} \mathrm{~d} z \int_{-\infty}^{\infty} \mathrm{d} x \delta(x) \int_{-\infty}^{\infty} \mathrm{d} y \delta(y)
$$

where $z_{1}=1\left(0,0, z_{1}\right)$ and $z_{2}=2\left(0,0, z_{2}\right)$. This description is used in Ref. [3].

Then, $\vec{r}$ means $r=|\vec{r}|$ because we set $z_{1}=0$ and $z_{2}=r$ in relative coordinate ( $r$ is the distance between $q(1)$ and $q^{\dagger}(2)$ ).

Then Equation (6) is expressed in relative coordinate as below.

$$
\begin{aligned}
i \frac{\partial}{\partial t} q(t ; r)= & -[i \vec{\alpha} \cdot \vec{\nabla}(r), q(t ; r)]+\beta m_{2} q(t ; r)-q(t ; r) \beta m_{1} \\
& -\frac{g^{2}}{2} \int_{0}^{r} \mathrm{~d} z q(t ; r-z)(r-z) q(t ; z) \\
& +\frac{g^{2}}{2} \int_{-\infty}^{t} \mathrm{~d} t^{\prime}(\vec{\alpha} \cdot \hat{r}) \delta\left(t-t^{\prime}\right) \int_{0}^{r} \mathrm{~d} z q\left(t^{\prime} ; r-z\right) q\left(t^{\prime} ; z\right)
\end{aligned}
$$

Except for the mass terms, Equation (11) was previously obtained in Ref. [3].

We decompose $q(r)$ to a Lorentz invariant description as follows.

$$
q(t ; r)=1 q_{0}(t ; r)+(-i \vec{\alpha} \cdot \hat{r}) q_{1}(t ; r)+\beta q_{2}(t ; r)+\beta(i \vec{\alpha} \cdot \hat{r}) q_{3}(t ; r)
$$

The following kinetic terms are derived after sandwiching $q_{v}(t ; r)$ with a vacuum state $\langle 0|$ and a physical state $|P\rangle$.

Unit matrix component: $-\frac{2}{r} \chi_{1}(t ; r)$

$(-i \vec{\alpha} \cdot \hat{r})$ component: 0

$\beta$ component: $-2 \frac{\partial}{\partial r} \chi_{3}(t ; r)-\frac{2}{r} \chi_{3}(t ; r)$

$\beta(i \vec{\alpha} \cdot \hat{r})$ component: $2 \frac{\partial}{\partial r} \chi_{2}(t ; r)$

The derivation of kinetic terms of $\beta$ and $\beta(i \vec{\alpha} \cdot \hat{r})$ components is shown in Ref. [17]. The derivation of Unit matrix and $(-i \vec{\alpha} \cdot \hat{r})$ components is given in Appendix A.

For an evaluation leading to the electric terms, we follow the argument in Ref. [3]. After sandwiching it with the vacuum state $\langle 0|$ and the physical state $|P\rangle$, the electric term becomes as follows.

$$
\begin{aligned}
& -\frac{g^{2}}{2}\left\langle 0\left|\int_{0}^{r} \mathrm{~d} z q(t ; r-z)(r-z) q(t ; z)\right| P\right\rangle \\
& =-\frac{g^{2}}{2}\left(\langle P| \int_{0}^{r} \mathrm{~d} z q^{\dagger}(t ; z)(r-z) q^{\dagger}(t ; r-z)\right)^{\dagger}
\end{aligned}
$$

In Ref. [3] we showed that the Hermitian conjugate of $q(1,2)$ in relative coordinates, i.e., $q(t ; r)$, is equal to taking $\hat{r} \rightarrow-\hat{r}$. The decomposition of the Hermitian conjugate of $q(t ; r)$ becomes

$$
q^{\dagger}(t ; r)=1 q_{0}^{\dagger}(t ; r)+(-i \vec{\alpha} \cdot \hat{r}) q_{1}^{\dagger}(t ; r)+\beta q_{2}^{\dagger}(t ; r)-\beta(i \vec{\alpha} \cdot \hat{r}) q_{3}^{\dagger}(t ; r)
$$


Equation (13) then becomes as follows.

$$
\begin{aligned}
\text { Eq.(13) }= & -\frac{g^{2}}{2}\left(\langle P | \int _ { 0 } ^ { r } \mathrm { d } z \left(1 q_{0}^{\dagger}(t ; z)+(-i \vec{\alpha} \cdot \hat{r}) q_{1}^{\dagger}(t ; z)+\beta q_{2}^{\dagger}(t ; z)\right.\right. \\
& \left.-\beta(i \vec{\alpha} \cdot \hat{r}) q_{3}^{\dagger}(t ; z)\right)(r-z)\left(1 q_{0}^{\dagger}(t: r-z)+(-i \vec{\alpha} \cdot \hat{r}) q_{1}^{\dagger}(t ; r-z)\right. \\
& \left.\left.+\beta q_{2}^{\dagger}(t ; r-z)-\beta(i \vec{\alpha} \cdot \hat{r}) q_{3}^{\dagger}(t ; r-z)\right)|0\rangle\right)^{\dagger}
\end{aligned}
$$

Here, we consider the contribution of each component. As an example, we show the detailed calculation of the $\beta$ component.

$$
\begin{aligned}
\beta \text { term }= & -\frac{g^{2}}{2}\left(\langle P | \beta \int _ { 0 } ^ { r } \mathrm { d } z ( r - z ) \left(q_{0}^{\dagger}(t ; z) q_{2}^{\dagger}(t ; r-z)+q_{2}^{\dagger}(t ; z) q_{0}^{\dagger}(t ; r-z)\right.\right. \\
& \left.\left.+q_{1}^{\dagger}(t ; z) q_{3}^{\dagger}(t ; r-z)-q_{3}^{\dagger}(t ; z) q_{1}^{\dagger}(t ; r-z)\right)|0\rangle\right)^{\dagger} \\
= & -\frac{g^{2}}{2}\left(\langle P | \beta \int _ { 0 } ^ { r } \mathrm { d } z ( r - z ) \left(q_{2}^{\dagger}(t ; r-z) q_{0}^{\dagger}(t ; z)+q_{0}^{\dagger}(t ; r-z) q_{2}^{\dagger}(t ; z)\right.\right. \\
& \left.\left.+q_{3}^{\dagger}(t ; r-z) q_{1}^{\dagger}(t ; z)-q_{1}^{\dagger}(t ; r-z) q_{3}^{\dagger}(t ; z)\right)|0\rangle\right)^{\dagger} \\
= & -\frac{g^{2}}{2} \beta^{\dagger}\left(\int_{0}^{r} \mathrm{~d} z\left\langle P\left|q_{3}^{\dagger}(t ; r-z)\right| 0\right\rangle\left\langle 0\left|q_{1}^{\dagger}(t ; z)\right| 0\right\rangle\right)^{\dagger} \\
= & -\frac{g^{2}}{2}\left(\langle P | \beta \int _ { 0 } ^ { r } \mathrm { d } z ( r - z ) \left(q_{2}^{\dagger}(t ; r-z)|0\rangle\langle 0| q_{0}^{\dagger}(t: z)\right.\right. \\
& +q_{0}^{\dagger}(t ; r-z)|0\rangle\left\langle 0\left|q_{2}^{\dagger}(t ; z)+q_{3}^{\dagger}(t ; r-z)\right| 0\right\rangle\langle 0| q_{1}^{\dagger}(t ; z) \\
& \left.\left.-q_{1}^{\dagger}(t ; r-z)|0\rangle\langle 0| q_{3}^{\dagger}(t ; z)\right)|0\rangle\right)^{\dagger} \\
= & \beta\left[-\frac{g^{2}}{2} \int_{0}^{r} \mathrm{~d} z\left\langle 0\left|q_{1}(t ; z)\right| 0\right\rangle\left\langle 0\left|q_{3}(t ; r-z)\right| P\right\rangle\right]
\end{aligned}
$$

In the second line of Equation (16), we commute fields because these are scalar quantities. In the third line, we insert a $|0\rangle\langle 0|$ term. Rigorously, this should be $1=\sum|n\rangle\langle n|$ where $\mathrm{n}$ denotes all states including the vacuum state. The expression $\langle 0|q(r)| P\rangle$ represents a real meson as a bound system but $\langle n|q(r)| P\rangle(n \neq 0)$ represents an unbound state such as $q-\bar{q}$ jet state (refer Ref. [3]). Thus we neglect all states except the vacuum state given by the $|0\rangle\langle 0|$ term.

In the fourth line, we use the condition from Ref. [3] that

$$
\left\langle 0\left|q_{0}(t ; r)\right| 0\right\rangle=0,\left\langle 0\left|q_{1}(t ; r)\right| 0\right\rangle=L_{1} \delta(r),\left\langle 0\left|q_{2}(r)\right| 0\right\rangle=0,\left\langle 0\left|q_{3}(r)\right| 0\right\rangle=0
$$

where the vacuum expectation value is $S(t ; r)=\langle 0|q(t ; r)| 0\rangle$.

The reason of this choice of conditions is given in Ref. [17].

Using $1^{\dagger}=1, \quad(-i \vec{\alpha} \cdot \hat{r})^{\dagger}=-(-i \vec{\alpha} \cdot \hat{r}), \quad \beta^{\dagger}=\beta, \quad(\beta(i \vec{\alpha} \cdot \hat{r}))^{\dagger}=\beta(i \vec{\alpha} \cdot \hat{r})$, we obtain the electric term as follows.

Unit matrix component: $\frac{g^{2} L_{1}}{2} r \chi_{1}(t ; r)$

$(-i \vec{\alpha} \cdot \hat{r})$ component: $\frac{g^{2} L_{1}}{2} r \chi_{0}(t ; r)$ 
B component: $-\frac{g^{2} L_{1}}{2} r \chi_{3}(t ; r)$

$\beta(i \vec{\alpha} \cdot \hat{r})$ component: $-\frac{g^{2} L_{1}}{2} r \chi_{2}(t ; r)$

Here, we use the definition of the amplitude given in Equation (3).

For the magnetic term, using the same argument as the electric term, we obtain:

Unit matrix component: $-i \frac{g^{2} L_{1}}{2} \int_{-\infty}^{t} \mathrm{~d} t^{\prime} \delta\left(t-t^{\prime}\right) \chi_{0}\left(t^{\prime} ; r\right)$

$(-i \vec{\alpha} \cdot \hat{r})$ component: $-i \frac{g^{2} L_{1}}{2} \int_{-\infty}^{t} \mathrm{~d} t^{\prime} \delta\left(t-t^{\prime}\right) \chi_{1}\left(t^{\prime} ; r\right)$

B component: $-i \frac{g^{2} L_{1}}{2} \int_{-\infty}^{t} \mathrm{~d} t^{\prime} \delta\left(t-t^{\prime}\right) \chi_{2}\left(t^{\prime} ; r\right)$

$\beta(i \vec{\alpha} \cdot \hat{r})$ component: $-i \frac{g^{2} L_{1}}{2} \int_{-\infty}^{t} \mathrm{~d} t^{\prime} \delta\left(t-t^{\prime}\right) \chi_{3}\left(t^{\prime} ; r\right)$

Remembering that we work in the center of mass of the system and in the rest frame in relative coordinates, $\chi(1,2)$ is expressed as follows.

$$
\chi(1,2)=\mathrm{e}^{-P_{0} t} \mathrm{e}^{i \vec{P} \cdot \vec{G}} \chi(r)=\mathrm{e}^{-i P_{0} t} \chi(r)=\chi(t ; r)
$$

Then, a time integral is carried out as shown in Ref. [3] and we obtain in the following.

$$
\int_{-\infty}^{t} \mathrm{~d} t^{\prime} \delta\left(t-t^{\prime}\right) \chi_{v}\left(t^{\prime} ; r\right)=\frac{\delta(0)}{-i P_{0}} \mathrm{e}^{-P_{0} t} \chi_{v}(r)
$$

In the case of the mass term, from Equation (11), after sandwiching $q_{v}(t ; r)$ by the vacuum state $\langle 0|$ and physical state $|P\rangle$, each component becomes the following.

Unit matrix component: $\left(m_{2}-m_{1}\right) \chi_{2}(t ; r)$

$(-i \vec{\alpha} \cdot \hat{r})$ component: $-\left(m_{2}+m_{1}\right) \chi_{3}(t ; r)$

B component: $\left(m_{2}-m_{1}\right) \chi_{0}(t ; r)$

$\beta(i \vec{\alpha} \cdot \hat{r})$ component: $-\left(m_{2}+m_{1}\right) \chi_{1}(t ; r)$

If we consider that these terms describe the mass terms of a meson particle, those of an anti-meson particle would be described as follows, because $q^{\dagger}(1,2)=q(2,1) \quad[3]$.

Unit matrix component: $\left(m_{1}-m_{2}\right) \chi_{2}(t ; r)$

$(-i \vec{\alpha} \cdot \hat{r})$ component: $-\left(m_{1}+m_{2}\right) \chi_{3}(t ; r)$

B component: $\left(m_{1}-m_{2}\right) \chi_{0}(t ; r)$

$\beta(i \vec{\alpha} \cdot \hat{r})$ component: $-\left(m_{1}+m_{2}\right) \chi_{1}(t ; r)$

Remembering that the masses of a particle and an anti-particle are the same, the mean value must be as below.

$$
\left.\frac{1}{2} \text { (mass of particle }+ \text { mass of antiparticle }\right)
$$

Then, the actual mass terms become the following. 
Unit matrix component: 0

$(-i \vec{\alpha} \cdot \hat{r})$ component: $-\left(m_{1}+m_{2}\right) \chi_{3}(t ; r)$

B component: 0

$\beta(i \vec{\alpha} \cdot \hat{r})$ component: $-\left(m_{1}+m_{2}\right) \chi_{1}(t ; r)$

After factoring out $\mathrm{e}^{-i P_{0} t}$, the equations of motion for the wave functions become as follows.

$$
\begin{gathered}
P_{0} \chi_{0}(r)=-\frac{2}{r} \chi_{1}(r)+\frac{g^{2} L_{1}}{2} r \chi_{1}(r)+\frac{g^{2} L_{1} \delta(0)}{2 P_{0}} \chi_{0}(r) \\
P_{0} \chi_{1}(r)=\frac{g^{2} L_{1}}{2} r \chi_{0}(r)+\frac{g^{2} L_{1} \delta(0)}{2 P_{0}} \chi_{1}(r)-\left(m_{1}+m_{2}\right) \chi_{3}(r) \\
P_{0} \chi_{2}(r)=-2 \frac{\partial}{\partial r} \chi_{3}(r)-\frac{2}{r} \chi_{3}(r)-\frac{g^{2} L_{1}}{2} r \chi_{3}(r)+\frac{g^{2} L_{1} \delta(0)}{2} \chi_{2}(r) \\
P_{0} \chi_{3}(r)=2 \frac{\partial}{\partial r} \chi_{2}(r)-\frac{g^{2} L_{1}}{2} r \chi_{2}(r)+\frac{g^{2} L_{1} \delta(0)}{2 P_{0}} \chi_{3}(r)-\left(m_{1}+m_{2}\right) \chi_{1}(r)
\end{gathered}
$$

Here we introduce new notation for $\overline{P_{0}}$.

$$
\overline{P_{0}}=P_{0}-\frac{g^{2} L_{1} \delta(0)}{2 P_{0}}
$$

Then, Equation (24) can be written as below.

$$
\chi_{0}(r)=\frac{1}{\overline{P_{0}}}\left(-\frac{2}{r} \chi_{1}(r)+\frac{g^{2} L_{1}}{2} \chi_{1}(r)\right)
$$

Substituting Equation (28) into Equation (25), $\chi_{1}(r)$ is expressed by $\chi_{3}(r)$ as the following.

$$
\chi_{1}(r)=-\frac{\overline{P_{0}}\left(m_{1}+m_{2}\right) \chi_{3}(r)}{\left(\bar{P}^{2}+g^{2} L_{1}\right)-\left(\frac{g^{2} L_{1}}{2} r\right)^{2}}
$$

From Equation (26), $\chi_{2}(r)$ can be expressed by $\chi_{3}(r)$ as follows.

$$
\chi_{2}(r)=-\frac{1}{\overline{P_{0}}}\left(2 \frac{\partial}{\partial r} \chi_{3}(r)+\frac{2}{r} \chi_{3}(r)+\frac{g^{2} L_{1}}{2} \chi_{3}(r)\right)
$$

Substitute Equation (30) into Equation (27) and using Equation (28), we obtain the equation for $\chi_{3}(r)$ as below.

$$
\begin{aligned}
\bar{P}^{2} \chi_{3}= & -4 \frac{\partial^{2} \chi_{3}}{\partial r^{2}}-\frac{4}{r} \frac{\partial \chi_{3}}{\partial r}+\frac{4}{r^{2}} \chi_{3}+\left(\frac{g^{2} L_{1}}{2}\right)^{2} r^{2} \chi_{3} \\
& +\frac{\bar{P}_{0}^{2}\left(m_{1}+m_{2}\right)^{2}}{\left(\bar{P}^{2}+g^{2} L_{1}\right)-\left(\frac{g^{2} L_{1}}{2} r\right)^{2}} \chi_{3}
\end{aligned}
$$

Starting with Equation (27) and using Equation (29) to express $\chi_{3}(r)$ as a function of $\chi_{2}(r)$, we then substitute into Equation (26) to obtain the following 
equation for $\chi_{2}(r)$

$$
\begin{aligned}
\bar{P}_{0}^{2} \chi_{2}= & -4 \frac{\partial^{2} \chi_{2}}{\partial r^{2}}-\frac{4}{r} \frac{\partial \chi_{2}}{\partial r}+4\left(\frac{g^{2} L_{1}}{2}\right) \chi_{2}+\left(\frac{g^{2} L_{1}}{2}\right)^{2} r^{2} \chi_{2} \\
& +\frac{\bar{P}^{2}\left(m_{1}+m_{2}\right)^{2}}{\left(\bar{P}^{2}+g^{2} L_{1}\right)-\left(\frac{g^{2} L_{1}}{2} r\right)^{2}} \chi_{2} \\
& +\frac{\left(m_{1}+m_{2}\right)^{2}\left(g^{2} L_{1}\right)\left(\frac{g^{2} L_{1}}{2} r\right)}{\left[\left(\bar{P}^{2}+g^{2} L_{1}\right)-\left(m_{1}+m_{2}\right)^{2}\right]^{2}}\left(-4 \frac{\partial \chi_{2}}{\partial r}+2\left(\frac{g^{2} L_{1}}{2}\right) r \chi_{2}\right)
\end{aligned}
$$

where

$$
\overline{P_{0}}=P_{0}-\frac{g^{2} L_{1} \delta(0)}{2 P_{0}}
$$

and $\chi_{3}$ and $\chi_{2}$ denote $\chi_{3}(r)$ and $\chi_{2}(r)$, respectively.

Here, we denote $H_{0}^{\left(\chi_{3}\right)}$ and $H_{0}^{\left(\chi_{2}\right)}$ as below.

$$
\begin{gathered}
H_{0}^{\left(\chi_{3}\right)}=-4 \frac{\partial^{2}}{\partial r^{2}}-\frac{4}{r} \frac{\partial}{\partial r}+\frac{4}{r^{2}}+\left(\frac{g^{2} L_{1}}{2}\right)^{2} r^{2} \\
H_{0}^{\left(\chi_{2}\right)}=-4 \frac{\partial^{2}}{\partial r^{2}}-\frac{4}{r} \frac{\partial}{\partial r}+4\left(\frac{g^{2} L_{1}}{2}\right)+\left(\frac{g^{2} L_{1}}{2}\right)^{2} r^{2}
\end{gathered}
$$

Equation (33) and Equation (34) are exactly the same Hamiltonian as the chiral limit case for $\chi_{3}(r)$ and $\chi_{2}(r)$, respectively, as shown in Ref. [3]. Thus, we consider $H_{0}^{\left(\chi_{3}\right)}$ and $H_{0}^{\left(\chi_{2}\right)}$ to be unperturbed Hamiltonians of Equation (31) and Equation (32), respectively. Then the remaining terms denoted as $H_{1}^{\left(\chi_{3}\right)}, H_{1}^{\left(\chi_{2}\right)(1)}$ and $H_{1}^{\left(\chi_{2}\right)(2)}$ are considered to be perturbed Hamiltonians of Equation (31) and Equation (32), respectively. These terms are expressed as below.

$$
\begin{gathered}
H_{1}^{\left(\chi_{3}\right)}=\frac{\bar{P}_{0}^{2}\left(m_{1}+m_{2}\right)^{2}}{\left(\bar{P}_{0}^{2}+g^{2} L_{1}\right)-\left(\frac{g^{2} L_{1}}{2} r\right)^{2}} \\
H_{1}^{\left(\chi_{2}\right)(1)}=\frac{\bar{P}_{0}^{2}\left(m_{1}+m_{2}\right)^{2}}{\left(\bar{P}^{2}+g^{2} L_{1}\right)-\left(\frac{g^{2} L_{1}}{2} r\right)^{2}} \\
H_{1}^{\left(\chi_{2}\right)(2)}=\frac{\left(m_{1}+m_{2}\right)^{2}\left(g^{2} L_{1}\right)\left(\frac{g^{2} L_{1}}{2} r\right)}{\left[\left(\bar{P}^{2}+g^{2} L_{1}\right)-\left(m_{1}+m_{2}\right)^{2}\right]^{2}}\left(-4 \frac{\partial}{\partial r}+2\left(\frac{g^{2} L_{1}}{2}\right) r\right)
\end{gathered}
$$

Then, we use the first order perturbation to evaluate the corrective light meson mass. For the $\chi_{3}(r)$ case, we use Equation (35) to determine the first order 
change in energy (mass) as follows.

$$
E_{1}^{\left(\chi_{3}\right)}=\left\langle n_{0}^{\left(\chi_{3}\right)}\left|H_{1}^{\left(\chi_{3}\right)}\right| n_{0}^{\left(\chi_{3}\right)}\right\rangle
$$

where $\left|n_{0}^{\left(\chi_{3}\right)}\right\rangle$ is the normalized wave function of the unperturbed Hamiltonian for $\chi_{3}(r)$.

As mentioned before, because the unperturbed Hamiltonian is the same as the Hamiltonian of the chiral limit case, the wave function of the unperturbed Hamiltonian for $\chi_{3}(r)$ is as follows.

$$
\chi_{3}(r)=\text { cont } r \exp \left(-\frac{g^{2} L_{1}}{8} r^{2}\right) F\left(1-\kappa^{\chi_{3}}, 2 ; \frac{g^{2} L_{1}}{4} r^{2}\right)
$$

where $\kappa^{\chi_{3}}$ is positive integer.

$F$ is the confluent hypergeometric series, as defined in Ref. [18].

$$
F(\alpha, \gamma ; Z)=\sum_{n=0}^{n} \frac{\alpha(\alpha+1) \cdots(\alpha+n-1)}{\gamma(\gamma+1) \cdots(\gamma+n-1)} \frac{z^{n}}{n !}
$$

The corresponding eigenvalue is the following, as given in Ref. [3].

$$
{\overline{P_{0}}}^{2}=4 g^{2} L_{1} \kappa^{\chi_{3}}
$$

Then, the normalized wave function of the unperturbed Hamiltonian $\left|n_{0}^{\left(\chi_{3}\right)}\right\rangle$ is expressed as the following.

$$
\left|n_{0}^{\left(\chi_{3}\right)}\right\rangle=\chi_{3}(r) /\left(4 \pi \int_{0}^{\infty} \mathrm{d} r r^{2} \chi_{3}(r)\right)
$$

The same argument used for $\chi_{3}(r)$ can be used to express $\chi_{2}(r)$, as below.

$$
\chi_{2}(r)=\text { const } \exp \left(-\frac{g^{2} L_{1}}{8} r^{2}\right) F\left(\frac{1}{2}-\kappa^{\chi_{2}}, 1 ; \frac{g^{2} L_{1}}{4} r^{2}\right)
$$

where $\kappa^{\chi_{2}}$ is a positive half integer.

The corresponding eigenvalue is the following.

$$
\bar{P}^{2}=4 g^{2} L_{1}\left(\kappa^{\chi_{2}}+\frac{1}{2}\right)
$$

Then, the normalized wave function $\left|n_{0}^{\chi_{2}}\right\rangle$ is expressed as below.

$$
\left|n_{0}^{\chi_{2}}\right\rangle=\chi_{2}(r) /\left(4 \pi \int_{0}^{\infty} \mathrm{d} r r^{2} \chi_{2}(r)\right)
$$

Note that the eigenvalue of $\chi_{3}(r)$ and $\chi_{2}(r)$ is the same for each corresponding meson, as shown in Ref. [3].

Then, using Equation (36) and Equation (37), the first order correction of the mass (energy) for $\chi_{2}(r)$ is evaluated as below.

$$
E_{1}^{\left(\chi_{2}\right)}=\left\langle n_{0}^{\chi_{2}}\left|H_{1}^{\left(\chi_{2}\right)(1)}\right| n_{0}^{\chi_{2}}\right\rangle+\left\langle n_{0}^{\chi_{2}}\left|H_{1}^{\left(\chi_{2}\right)(2)}\right| n_{0}^{\chi_{2}}\right\rangle
$$

\section{Evaluation}

In this section, we show how to perform the first order perturbation. Before proceeding with this argument, we insist on the fact that pion solutions are un- 
changed even though the masses of $\mathrm{u}, \mathrm{d}$, and $\mathrm{s}$ quarks become nonzero. This is obvious when we substitute the following pion wave functions with an eigenvalue of $P_{0}^{2}=\frac{g^{2} L_{1} \delta(0)}{2}=m_{\pi}^{2}$ into Equations (24)-(27).

$$
\begin{gathered}
\chi_{0}^{(\pi)}(r)=\text { const } \frac{2\left(m_{1}+m_{2}\right)}{g^{2} L_{1}} \frac{1}{r^{2}} \mathrm{e}^{-\frac{g^{2} L_{1}}{8} r^{2}} \\
\chi_{1}^{(\pi)}(r)=0 \\
\chi_{2}^{(\pi)}(r)=0 \\
\chi_{3}^{(\pi)}(r)=\text { const } \frac{1}{r} \mathrm{e}^{-\frac{g^{2} L_{1}}{8} r^{2}}
\end{gathered}
$$

These solutions exactly satisfy Equations (24)-(27) with an eigenvalue of $P_{0}^{2}=\frac{g^{2} L_{1} \delta(0)}{2}=m_{\pi}^{2}$. Thus, pion mass is unchanged even though the constituent quark mass becomes nonzero. Next we show how to evaluate the first order correction of mass for light mesons except pions. In this procession, we also consider the contribution of the spin effect for vector mesons. From Equation (38), Equation (39), Equation (42), Equation (43), Equation (45), and Equation (46), the essential integrations we must perform are as follows.

$$
\begin{gathered}
I_{1}^{\left(\chi_{3}\right)}=\int_{0}^{\infty} \mathrm{d} r \mathrm{e}^{-\frac{g^{2} L_{1}}{4} r^{2}}\left(\frac{g^{2} L_{1}}{4}\right)^{2 n} r^{4 n+4} \frac{\bar{P}_{0}^{2}\left(m_{1}+m_{2}\right)^{2}}{\left(\bar{P}_{0}^{2}+g^{2} L_{1}\right)-\left(\frac{g^{2} L_{1}}{2} r\right)^{2}} \\
I_{1}^{\left(\chi_{2}\right)(1)}=\int_{0}^{\infty} \mathrm{d} r \mathrm{e}^{-\frac{g^{2} L_{1}}{4} r^{2}}\left(\frac{g^{2} L_{1}}{4}\right)^{2 n} r^{4 n+2} \frac{\bar{P}_{0}^{2}\left(m_{1}+m_{2}\right)^{2}}{\left(\bar{P}^{2}+g^{2} L_{1}\right)-\left(\frac{g^{2} L_{1}}{2} r\right)^{2}} \\
I_{1}^{\left(\chi_{2}\right)(2)}=\int_{0}^{\infty} \mathrm{d} r \mathrm{e}^{-\frac{g^{2} L_{1}}{4} r^{2}}\left(\frac{g^{2} L_{1}}{4}\right)^{2 n} r^{4 n+2} \frac{\frac{3 n+2}{2 n} 4\left(m_{1}+m_{2}\right)^{2}\left(g^{2} L_{1}\right)\left(\frac{g^{2} L_{1}}{2} r\right)^{2}}{\left[\left(\bar{P}^{2}+g^{2} L_{1}\right)-\left(m_{1}+m_{2}\right)^{2}\right]^{2}} \\
\text { where } n=\kappa^{\chi_{3}}-1=\left(\kappa^{\chi_{2}}+\frac{1}{2}\right)-1 \text { and } \bar{P}_{0}^{2}=4 \kappa^{\chi_{3}} g^{2} L_{1}=4\left(\kappa^{\chi_{2}}+\frac{1}{2}\right) g^{2} L_{1} .
\end{gathered}
$$

Equation (53) is obtained from the following consideration. Remembering that the expression of $\chi_{2}(r)$ is given in Equation (43), the factor $-4 \frac{\partial \chi_{2}}{\partial r}+2\left(\frac{g^{2} L_{1}}{2}\right) r \chi_{2}$ can be evaluated by changing the variable $z=\frac{g^{2} L_{1}}{4} r^{2}$ as follows.

$$
\left(-4 \frac{\partial}{\partial r}+2\left(\frac{g^{2} L_{1}}{2}\right) r\right) \chi_{2}(r)=(-4)\left(\frac{g^{2} L_{1}}{2} r\right)\left[\frac{\partial}{\partial z}-\frac{1}{2}\right]\left[\mathrm{e}^{-\frac{z}{2}} F\left(\frac{1}{2}-\kappa^{\chi_{2}}, 1 ; z\right)\right]
$$

Using the following formula from Ref. [18] for the derivative of the confluent 
hypergeometric series,

$$
\frac{\partial}{\partial z} F(\alpha, \gamma ; z)=F(\alpha, \gamma ; z)+\left(\frac{\alpha}{\gamma}-1\right) F(\alpha, \gamma+1 ; z)
$$

We obtain Equation (54) as follows.

Eq. (54)

$$
\begin{aligned}
& =(-4)\left(\frac{g^{2} L_{1}}{2} r\right) \mathrm{e}^{-\frac{z}{2}}\left(-1+\frac{1}{2} F\left(\frac{1}{2}-\kappa^{\chi_{2}}, 1 ; z\right)-\left(\frac{1}{2}+\kappa^{\chi_{2}}\right) F\left(\frac{1}{2}-\kappa^{\chi_{2}}, 2 ; z\right)\right) \\
& =\frac{3 n+2}{2 n} 4\left(\frac{g^{2} L_{1}}{2} r\right) \mathrm{e}^{-\frac{z}{2}} F\left(\frac{1}{2}-\kappa^{\chi_{2}}, 1 ; z\right)
\end{aligned}
$$

For the second line, we use the fact that $n=\kappa^{\chi_{2}}-\frac{1}{2}$ and obvious result that

$$
(n+1) F(1-n, 2 ; z)=\frac{n+1}{n} F(1-n, 1 ; z) \text {. }
$$

Equation (55) is satisfied under the condition that $n$ is a positive integer, that is, $\kappa^{\chi_{2}}$ is a half integer larger than $\frac{3}{2}$. For the $n=0\left(\kappa^{\chi_{2}}=\frac{1}{2}\right)$ case, we use Equation (37) to evaluate $\left\langle n_{0}^{\chi_{2}}\left|H_{1}^{\left(\chi_{2}\right)(2)}\right| n_{0}^{\chi_{2}}\right\rangle$. Either way, we neglect this term, for the following reasons.

Remembering that $\frac{g^{2} L_{1} \delta(0)}{2}=m_{\pi}^{2}$ and that $\delta(0)$ is considered as a renormalization factor, we can conclude that $\delta(0)=1$. This is because, under this setting, we obtain the plausible light meson mass spectra for the chiral limit case and the equal time commutation relation gives also $\delta(0)=1$. Thus $g^{2} L_{1}=2 m_{\pi}^{2}$. Because the integral of Equation (52) has a simple pole at $r=\frac{2}{g^{2} L_{1}} \sqrt{{\overline{P_{0}}}^{2}+g^{2} L_{1}}$ and that of Equation (53) has order 2 pole at $r=\frac{2}{g^{2} L_{1}} \sqrt{{\overline{P_{0}}}^{2}+g^{2} L_{1}-\left(m_{1}+m_{2}\right)^{2}}$, rough estimation shows that Equation (53) is smaller than Equation (52) by the factor $g^{2} L_{1}$. Thus, we can neglect $\left\langle H_{1}^{\left(\chi_{2}\right)(2)}\right\rangle$ compared to $\left\langle H_{1}^{\left(\chi_{2}\right)(1)}\right\rangle$. From now on, to proceed the evaluation of Equation (51) and Equation (52), we use the notation $\overline{P_{0}^{\prime}}=\sqrt{\bar{P}_{0}^{2}}+g^{2} L_{1}$. Then, Equation (51) is evaluated as below.

$$
\begin{aligned}
I_{1}^{\left(\chi_{3}\right)} & =\left(\frac{g^{2} L_{1}}{4}\right)^{2 n} \int_{0}^{\infty} \mathrm{d} r \mathrm{e}^{-\frac{g^{2} L_{1}}{4} r^{2}} r^{4 n+4} \frac{{\overline{P_{0}}}^{2}\left(m_{1}+m_{2}\right)^{2}}{\left(\overline{P_{0}^{\prime}}-\frac{g^{2} L_{1}}{2} r\right)\left(\overline{P_{0}^{\prime}}+\frac{g^{2} L_{1}}{2} r\right)} \\
& =\left(\frac{g^{2} L_{1}}{4}\right)^{2 n} \int_{0}^{\infty} \mathrm{d} r \mathrm{e}^{-\frac{g^{2} L_{1}}{4} r^{2}} r^{4 n+4} \frac{1}{2 \overline{P_{0}^{\prime}}}\left(\frac{1}{\overline{P_{0}^{\prime}}-g^{2} L_{1}}+\frac{1}{\overline{P_{0}^{\prime}}+g^{2} L_{1}}\right) \bar{P}_{0}^{2}\left(m_{1}+m_{2}\right)^{2}
\end{aligned}
$$

For the first term, changing the variable to $z=\overline{P_{0}^{\prime}}-\frac{g^{2} L_{1}}{2} r$ yields the following. 


$$
\begin{aligned}
& I_{1 \text { (first) }}^{\left(\chi_{3}\right)}=\left(\frac{g^{2} L_{1}}{4}\right)^{2 n} \frac{1}{2 \overline{P_{0}^{\prime}}} \int_{-\infty}^{\overline{P_{0}^{\prime}}} \mathrm{d} z \frac{1}{\left(\frac{g^{2} L_{1}}{2}\right)} \exp \left(-\frac{\left(\overline{P_{0}^{\prime}}-z\right)^{2}}{g^{2} L_{1}}\right) \\
& \times\left(\frac{\overline{P_{0}^{\prime}}-z}{\frac{g^{2} L_{1}}{2}}\right)^{4 n+4} \frac{1}{z} \bar{P}^{2}\left(m_{1}+m_{2}\right)^{2} \\
& =\frac{1}{2 \overline{P_{0}^{\prime}}} \frac{1}{2^{2 n}} \frac{1}{\left(\frac{g^{2} L_{1}}{2}\right)^{2 n+5}} \int_{-\infty}^{\overline{P_{0}^{\prime}}} \mathrm{d} z \frac{\exp \left(-\frac{\left(z-\overline{P_{0}^{\prime}}\right)^{2}}{g^{2} L_{1}}\right)}{z} \\
& \times \sum_{s=0}^{4 n+4}{ }_{4 n+4} C_{s}(-z)^{s}{\overline{P_{0}}}^{\prime 4 n+4-s}{\overline{P_{0}}}^{2}\left(m_{1}+m_{2}\right)^{2}
\end{aligned}
$$

For the second term, changing the variable to $z=\overline{P_{0}^{\prime}}+\frac{g^{2} L_{1}}{2}$ leads to the following.

$$
\begin{aligned}
I_{1(\text { second })}^{\left(\chi_{3}\right)}= & \frac{1}{2 \overline{P_{0}^{\prime}}} \frac{1}{2^{2 n}} \frac{1}{\left(\frac{g^{2} L_{1}}{2}\right)^{2 n+5}} \int_{\overline{P_{0}^{\prime}}}^{\infty} \mathrm{d} z \frac{\exp \left(-\frac{\left(z-\overline{P_{0}^{\prime}}\right)^{2}}{g^{2} L_{1}}\right)}{z} \\
& \times \sum_{s=0}^{4 n+4}{ }_{4 n+4} C_{s}(-z)^{s}{\overline{P_{0}^{\prime}}}^{4 n+4-s}{\overline{P_{0}}}^{2}\left(m_{1}+m_{2}\right)^{2}
\end{aligned}
$$

Here we use the fact that $\left(z-\overline{P_{0}^{\prime}}\right)^{4 n+4}=\left(-z+\overline{P_{0}^{\prime}}\right)^{4 n+4}$.

Then, $I_{1}^{\left(\chi_{3}\right)}$ is expressed as

$$
\begin{aligned}
& I_{1}^{\left(\chi_{3}\right)}= I_{1 \text { (first) }}^{\left(\chi_{3}\right)}+I_{1 \text { (scond) }}^{\left(\chi_{3}\right)} \\
&= \frac{1}{2 \overline{P_{0}^{\prime}}} \frac{1}{2^{2 n}} \frac{1}{\left(\frac{g^{2} L_{1}}{2}\right)^{2 n+5}} \int_{-\infty}^{\infty} \mathrm{d} z \frac{\exp \left(-\frac{\left(z-\overline{P_{0}^{\prime}}\right)^{2}}{g^{2} L_{1}}\right)}{z} \\
& \times \sum_{s=0}^{4 n+4}{ }_{4 n+4} C_{s}(-z)^{s}{\overline{P_{0}}}^{\prime 4 n+4-s} \bar{P}_{0}^{2}\left(m_{1}+m_{2}\right)^{2} \\
&= \frac{1}{2} \frac{1}{2^{2 n}} \frac{1}{\left(\frac{g^{2} L_{1}}{2}\right)^{\frac{5}{2}}} \int_{-\infty}^{\infty} \mathrm{d}\left(\frac{z}{\sqrt{\frac{g^{2} L_{1}}{2}}}\right)^{2}\left(-\frac{1}{2}\left(\frac{z}{\sqrt{\frac{g^{2} L_{1}}{2}}}-\frac{\overline{P_{0}^{\prime}}}{\left.\left.\sqrt{\frac{g^{2} L_{1}}{2}}\right)^{2}\right)}\right.\right. \\
& \frac{z}{\sqrt{\frac{g^{2} L_{1}}{2}}}
\end{aligned}
$$




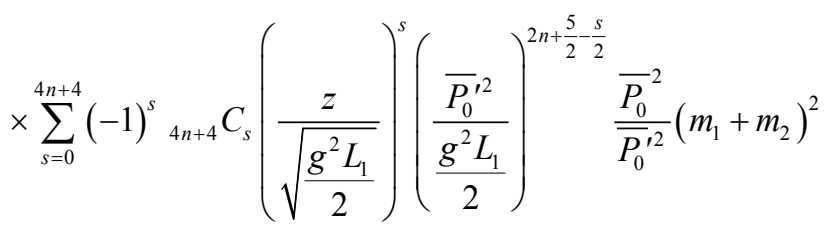

Remembering the fact that $\bar{P}_{0}^{2}=4 \kappa^{\chi_{3}} g^{2} L_{1},{\overline{P_{0}}}^{2}=\bar{P}_{0}^{2}+g^{2} L_{1}$ and $\kappa^{\chi_{3}}$ is positive integer, $\frac{\overline{P_{0}}}{\sqrt{\frac{g^{2} L_{1}}{2}}}, \frac{\overline{P_{0}^{\prime}}}{\sqrt{\frac{g^{2} L_{1}}{2}}}, \frac{z}{\sqrt{\frac{g^{2} L_{1}}{2}}}$ are dimensionless. Thus, the dimension of $I_{1}^{\left(\chi_{3}\right)}$ is $\frac{1}{\left(g^{2} L_{1}\right)^{\frac{5}{2}}} \times(\text { mass })^{2}$. Because $\left|n_{0}^{\chi_{3}}\right\rangle$ is a normalized wave $\left(\frac{g^{2} L_{1}}{2}\right)^{\frac{5}{2}}$

function, to evaluate $\left\langle n_{0}^{\chi_{3}}\left|H_{1}^{\left(\chi_{3}\right)}\right| n_{0}^{\chi_{3}}\right\rangle$, we must consider the denominator of Equation (43). The essential integral of this denominator is expressed as

$$
\begin{aligned}
I_{1(d e n)}^{\left(\chi_{3}\right)} & =\left(\frac{g^{2} L_{1}}{4}\right)^{2 n} \int_{0}^{\infty} \mathrm{d} r r^{4 n+4} \mathrm{e}^{-\frac{g^{2} L_{1} r^{2}}{4}}=\frac{1}{\left(\frac{g^{2} L_{1}}{4}\right)^{\frac{5}{2}}} \frac{1}{2} \Gamma\left(2 n+\frac{5}{2}\right) \\
& =\frac{2^{\frac{5}{2}}}{\left(\frac{g^{2} L_{1}}{2}\right)^{\frac{5}{2}}} \frac{1}{2} \Gamma\left(2 n+\frac{5}{2}\right)
\end{aligned}
$$

Notice that the factor $\left(\frac{g^{2} L_{1}}{2}\right)^{-\frac{5}{2}}$ is cancelled out so that the dimension of $E_{1}^{\left(\chi_{3}\right)}=\left\langle H_{1}^{\left(\chi_{3}\right)}\right\rangle$ is (mass) $)^{2}$ (i.e., energy).

Changing the variable to $z^{\prime}=\frac{z}{\sqrt{\frac{g^{2} L_{1}}{2}}}$, Equation (59) can be expressed as below.

$$
\begin{aligned}
I_{1}^{\left(\chi_{3}\right)}= & \frac{1}{2} \frac{1}{2^{2 n}} \frac{1}{\left(\frac{g^{2} L_{1}}{2}\right)^{\frac{5}{2}}} \int_{-\infty}^{\infty} \mathrm{d} z^{\prime} \frac{\exp \left(-\frac{1}{2}\left(z^{\prime}-\frac{\overline{P_{0}^{\prime}}}{\sqrt{\frac{g^{2} L_{1}}{2}}}\right)^{2}\right)}{z^{\prime}} \\
& \times \sum_{s=0}^{4 n+4}(-1)^{s}{ }_{4 n+4} C_{s} z^{\prime s}\left(\frac{\overline{P_{0}^{\prime 2}}}{\frac{g^{2} L_{1}}{2}}\right)^{2 n+\frac{5}{2}-\frac{s}{2}} \frac{{\overline{P_{0}}}^{2}}{{\overline{P_{0}^{\prime 2}}}^{\prime 2}\left(m_{1}+m_{2}\right)^{2}}
\end{aligned}
$$

Note that $z^{\prime}$ is dimensionless. From Equation (52), the difference of integral of 
$I_{1}^{\left(\chi_{2}\right)(1)}$ and the integral of $I_{1}^{\left(\chi_{3}\right)}$ is $r^{4 n+2}$ instead of $r^{4 n+4}$. Thus, using the same argument as for $I_{1}^{\left(\chi_{3}\right)}, I_{1}^{\left(\chi_{2}\right)(1)}$ becomes the following.

$$
\begin{aligned}
I_{1}^{\left(\chi_{2}\right)(1)}= & \frac{1}{2} \frac{1}{2^{2 n}} \frac{1}{\left(\frac{g^{2} L_{1}}{2}\right)^{\frac{3}{2}}} \int_{-\infty}^{\infty} \mathrm{d} z \frac{\exp \left(-\frac{1}{2}\left(z-\frac{\overline{P_{0}^{\prime}}}{\sqrt{\frac{g^{2} L_{1}}{2}}}\right)^{2}\right)}{z} \\
& \times \sum_{s=0}^{4 n+2}(-1)^{s}{ }_{4 n+2} C_{s} z^{s}\left(\frac{\overline{P_{0}^{\prime 2}}}{\frac{g^{2} L_{1}}{2}}\right)^{2 n+\frac{3}{2}-\frac{s}{2}} \frac{\bar{P}_{0}^{2}}{{\overline{P_{0}^{\prime 2}}}^{2}}\left(m_{1}+m_{2}\right)^{2}
\end{aligned}
$$

Here, we use the notation $z$ instead of $z$ ' We use this integral notation from here on. The essential integral in the denominator of Equation (45) is expressed as below.

$$
\begin{aligned}
I_{1(d e n)}^{\left(\chi_{2}\right)(1)} & =\left(\frac{g^{2} L_{1}}{4}\right)^{2 n} \int_{0}^{\infty} \mathrm{d} r r^{4 n+2} \mathrm{e}^{-\frac{g^{2} L_{1}}{4} r^{2}}=\frac{1}{\left(\frac{g^{2} L_{1}}{4}\right)^{\frac{3}{2}}} \frac{1}{2} \Gamma\left(2 n+\frac{3}{2}\right) \\
& =\frac{2^{\frac{3}{2}}}{\left(\frac{g^{2} L_{1}}{2}\right)^{\frac{3}{2}}} \frac{1}{2} \Gamma\left(2 n+\frac{3}{2}\right)
\end{aligned}
$$

Then, the dimension of $E_{1}^{\left(\chi_{2}\right)}=\left\langle n_{0}^{\chi_{2}}\left|H_{1}^{\left(\chi_{2}\right)(1)}\right| n_{0}^{\chi_{2}}\right\rangle$ is again (mass) ${ }^{2}$ (i.e. energy).

The Integral part of Equation (61) is described as the following.

$$
\begin{aligned}
J_{1}^{\left(\chi_{3}\right)}=\int_{-\infty}^{\infty} \mathrm{d} z \frac{\exp \left(-\frac{1}{2}\left(z-\frac{\overline{P_{0}^{\prime}}}{\sqrt{\frac{g^{2} L_{1}}{2}}}\right)^{2}\right)}{z}\left(\frac{\overline{P_{0}^{\prime 2}}}{\frac{g^{2} L_{1}}{2}}\right)^{2 n+\frac{5}{2}} \\
+\int_{-\infty}^{\infty} \mathrm{d} z \exp \left(-\frac{1}{2}\left(z-\frac{\overline{P_{0}^{\prime}}}{\sqrt{\frac{g^{2} L_{1}}{2}}}\right)^{2}\right)_{\sum_{\bar{s}=0}^{4 n+3}(-1)^{\bar{s}+1}{ }_{4 n+4} C_{\bar{s}+1} z^{\bar{s}}\left(\frac{\overline{P_{0}^{\prime 2}}}{\frac{g^{2} L_{1}}{2}}\right)^{2 n+2-\frac{\bar{s}}{2}}}
\end{aligned}
$$

From this description, we can notice the obvious fact that the main contribution of $J_{1}^{\left(\chi_{3}\right)}$ comes from the first term because the integral appears to have a singularity. Thus, we must evaluate this integral carefully.

To perform the integration of the first term, we modify it as follows. 


$$
\begin{aligned}
T_{1} & =\int_{-\infty}^{\infty} \mathrm{d} z \frac{1}{z} \exp \left(-\frac{1}{2}\left(z-\frac{\overline{P_{0}^{\prime}}}{\sqrt{\frac{g^{2} L_{1}}{2}}}\right)^{2}\right) \\
& =\int_{-\infty}^{0} \mathrm{~d} z \frac{1}{z} \exp \left(-\frac{1}{2}\left(z-\frac{\overline{P_{0}^{\prime}}}{\sqrt{\frac{g^{2} L_{1}}{2}}}\right)^{2}\right)+\int_{0}^{\infty} \mathrm{d} z \frac{1}{z} \exp \left(-\frac{1}{2}\left(z-\frac{\overline{P_{0}^{\prime}}}{\sqrt{\frac{g^{2} L_{1}}{2}}}\right)^{2}\right)
\end{aligned}
$$

After changing the variable with $z=-z^{\prime}$, the first term becomes as follows.

$$
\begin{aligned}
T_{1 \text { (first) }} & =-\int_{0}^{\infty} \mathrm{d} z^{\prime} \frac{1}{z^{\prime}} \exp \left(-\frac{1}{2}\left(z^{\prime}+\frac{\overline{P_{0}^{\prime}}}{\sqrt{\frac{g^{2} L_{1}}{2}}}\right)^{2}\right) \\
& =\exp \left(-\frac{\overline{P_{0}^{\prime 2}}}{g^{2} L_{1}}\right) \int_{0}^{\infty} \mathrm{d} z^{\prime} \frac{1}{z^{\prime}}(-1) \exp \left(-\frac{z^{\prime 2}}{2}-\frac{\overline{P_{0}^{\prime} z^{\prime}}}{\sqrt{\frac{g^{2} L_{1}}{2}}}\right)
\end{aligned}
$$

The second term becomes the following.

$$
T_{1 \text { (second) }}=\exp \left(-\frac{\overline{P_{0}^{\prime 2}}}{g^{2} L_{1}}\right) \int_{0}^{\infty} \mathrm{d} z \frac{1}{z} \exp \left(-\frac{z^{2}}{2}+\frac{\overline{P_{0}^{\prime} z}}{\sqrt{\frac{g^{2} L_{1}}{2}}}\right)
$$

Thus, integral $T_{1}$ is expressed as below.

$$
\begin{aligned}
T_{1} & =T_{1 \text { (first) }}+T_{1 \text { (second) }} \\
& =\exp \left(-\frac{\overline{P_{0}^{\prime 2}}}{g^{2} L_{1}}\right) \int_{0}^{\infty} \mathrm{d} z \frac{1}{z} \exp \left(-\frac{z^{2}}{2}\right)\left(\exp \left(\frac{\overline{P_{0}^{\prime} z}}{\sqrt{\frac{g^{2} L_{1}}{2}}}\right)-\exp \left(-\frac{\overline{P_{0}^{\prime} z}}{\sqrt{\frac{g^{2} L_{1}}{2}}}\right)\right) \\
& =\exp \left(-\frac{\overline{P_{0}^{\prime 2}}}{g^{2} L_{1}}\right) \int_{0}^{\infty} \mathrm{d} z \exp \left(-\frac{z^{2}}{2}\right) \frac{\left(\frac{\overline{P_{0}^{\prime} z}}{\sqrt{\frac{g^{2} L_{1}}{2}}}\right)}{z}
\end{aligned}
$$

Notice that Equation (68) does not have singularity. When $\bar{P}_{0}^{\prime} / \sqrt{\frac{g^{2} L_{1}}{2}}$ is sufficiently large, $T_{1}$ becomes as follows.

$$
T_{1} \approx \sqrt{\pi} \exp \left(-\frac{\overline{P_{0}^{\prime 2}}}{g^{2} L_{1}}\right)
$$

The derivation of this form is shown in Appendix B. 
In this paper, we use this expression for the calculation of correction masses except for the case of a kaon, an $f^{0}$ meson and an $\eta$ meson. For the kaon and $f^{0}$ meson cases, we use the exact representations of the integral and the second term of Equation (64). This is given in Appendix C. For the $\eta$ meson case, we use the exact representation of Equation (68) and calculate only the principal part of the second term of Equation (64). For the second terms of Equation (64), denoted by $T_{2}^{\left(\chi_{3}\right)}$, we use the following integral formula [19].

$$
\int_{-\infty}^{\infty} \mathrm{d} x x^{n} \exp \left(-(x-b)^{2}\right)=(2 i)^{-n} \sqrt{\pi} H_{n}(i b)
$$

where $H_{n}(x)=\sum_{r=0}^{\left[\frac{n}{2}\right]}(-1)^{r}(2 r-1) ! !{ }_{n} C_{2 r} x^{n-2 r} \quad$ (Hermite function).

Then, the second term of Equation (64) is expressed as follows.

$$
T_{2}^{\left(\chi_{3}\right)}=\sum_{\bar{s}=0}^{4 n+3}(-1)^{\bar{s}+1} 2^{\frac{\bar{s}+1}{2}}\left(\frac{\overline{P_{0}^{\prime 2}}}{\frac{g^{2} L_{1}}{2}}\right)^{2 n+2-\frac{\bar{s}}{2}}(2 i)^{-\bar{s}} \sqrt{\pi} H_{\bar{s}}\left(i \frac{\overline{P_{0}^{\prime}}}{\sqrt{g^{2} L_{1}}}\right)
$$

We neglect the $T_{2}^{\left(\chi_{3}\right)}$ terms because they are small compared to the $T_{1}$ term multiplied by $\left(\frac{\overline{P_{0}^{\prime 2}}}{\frac{g^{2} L_{1}}{2}}\right)^{2 n+\frac{3}{2}}$ when $\frac{\overline{P_{0}^{\prime}}}{\sqrt{\frac{g^{2} L_{1}}{2}}}$ becomes large. For the $\chi_{2}$ case, we use the same argument because the only difference is the exponent of $\frac{\overline{P_{0}^{\prime 2}}}{\frac{g^{2} L_{1}}{2}}$

term and the end term of summation.

\section{Results}

To determine masses of $\mathrm{u}, \mathrm{d}$, and s quarks, we use the evaluation of the masses of $\kappa^{+}\left(\kappa^{-}\right)$and $\kappa^{0}\left(\overline{\kappa^{0}}\right)$ because the constituent quarks of $\kappa^{+}\left(\kappa^{-}\right)$is $u \bar{s}(\bar{u} s)$ and those of $\kappa^{0}\left(\kappa^{0}\right)$ are $d \bar{s}(\overline{d s})$. The quantities of $\left\langle H_{i}^{\left(\chi_{3}\right)}\right\rangle /\left(m_{1}+m_{2}\right)^{2}$ and $\left\langle H_{1}^{\left(\chi_{2}\right)}\right\rangle /\left(m_{1}+m_{2}\right)^{2}$ are calculated by using the $f^{0}$ meson wave functions obtained in Equation (39) with $\kappa^{\chi_{3}}=1$ for $\chi_{3}$ and Equation (43) with $\kappa^{\chi_{2}}=\frac{1}{2}$ for $\chi_{2}$. The reasoning for this is that $f^{0}$ meson appears always appears with a kaon and that kaon mass is close to $f^{0}$ meson mass. Table 1 shows the obtained masses of $\mathrm{u}, \mathrm{d}$, and $\mathrm{s}$ quarks in the process of kaon mass evaluation. To evaluate each mass term, we use quark and antiquark constituents. For example, the mass term of $\kappa^{+}\left(\kappa^{-}\right)$is $\left(m_{u}+m_{s}\right)^{2}$ because we consider that the quark and antiquark masses are the same.

For the $\eta$ and $\eta^{\prime}$ mesons case, we evaluate the mass term as below.

$$
\eta:\left[\left(m_{u}+m_{u}\right)^{2}+\left(m_{d}+m_{d}\right)^{2}\right] \cos \theta-\left[m_{s}+m_{s}\right]^{2} \sin \theta
$$


Table 1. Quark masses.

\begin{tabular}{cccc}
\hline quark & $\mathrm{u}$ & $\mathrm{d}$ & $\mathrm{s}$ \\
\hline $\begin{array}{c}\text { Estimated mas } \\
(\mathrm{MeV})\end{array}$ & 1.12 & 3.56 & 62 \\
$\begin{array}{c}\text { Estimated mass } \\
(\text { Particle Data } \\
\text { Group) }(\mathrm{MeV})[20]\end{array}$ & $2.16_{-0.47}^{+0.49}$ & $4.67_{-0.17}^{+0.48}$ & $93_{-5}^{+11}$ \\
\hline
\end{tabular}

$$
\eta^{\prime}:\left[\left(m_{u}+m_{u}\right)^{2}+\left(m_{d}+m_{d}\right)^{2}\right] \cos \theta+\left[m_{s}+m_{s}\right]^{2} \sin \theta
$$

This is because the quark and antiquark constituents are as follows.

$$
\begin{gathered}
\eta:(u \bar{u}+d \bar{d}) \cos \theta-s \bar{s} \sin \theta \\
\eta^{\prime}:(u \bar{u}+d \bar{d}) \cos \theta+s \bar{s} \sin \theta
\end{gathered}
$$

For the $\rho$ meson and $\omega$ meson cases, the mass terms are expressed as below.

$$
\begin{gathered}
\rho^{+}\left(\rho^{-}\right):\left(m_{u}+m_{d}\right)^{2} \\
\rho^{0}:\left(m_{u}+m_{u}\right)^{2} \cos \theta-\left(m_{d}+m_{d}\right)^{2} \sin \theta \\
\omega^{0}:\left(m_{u}+m_{u}\right)^{2} \cos \theta+\left(m_{d}+m_{d}\right)^{2} \sin \theta
\end{gathered}
$$

These reflect the following quark and antiquark constitutions.

$$
\begin{gathered}
\rho^{+}\left(\rho^{-}\right): u \bar{d}(\bar{u} d) \\
\rho^{0}: u \bar{u} \cos \theta-d \bar{d} \sin \theta \\
\text { meson } \omega^{0}: u \bar{u} \cos \theta+d \bar{d} \sin \theta
\end{gathered}
$$

Because its constituent quark and antiquark are $s \bar{s}$, the mass term for a $\phi$ meson becomes the following.

$$
\phi:\left(m_{s}+m_{s}\right)^{2}
$$

For the vector meson, we have to consider the contribution of a spin effect.

To do this we define the spin contribution as below.

$$
(-1)^{Q+\bar{Q}^{+1}} \alpha_{s} \frac{1}{m_{q} m_{\bar{q}}}\left[\left\langle H_{1}^{\left(\chi_{3}\right)}\right\rangle+\left\langle H_{1}^{\left(\chi_{2}\right)}\right\rangle\right]
$$

where $Q$ and $\bar{Q}$ denote the charges of a quark and an antiquark, respectively. In addition, $\alpha_{s}$ is a spin parameter, $m_{q}$ and $m_{\bar{q}}$ denote masses of a quark and an antiquark, respectively. This description of spin contribution is based on that of Choi et al. [2].

The total perturbative energy $\left((\text { mass })^{2}\right.$ correction $\left.)\right)$ can then be described as below.

$$
E_{1(\text { pert })}=\left(1+\delta_{S 1}(-1)^{Q+\bar{Q}+1} \alpha_{S} \frac{1}{m_{q} m_{\bar{q}}}\right)\left[\left\langle H_{1}^{\left(\chi_{3}\right)}\right\rangle+\left\langle H_{1}^{\left(\chi_{2}\right)}\right\rangle\right]
$$


where $\delta_{S 1}$ is the Kronecker delta, which becomes 1 when a meson spin is 1 and it becomes 0 otherwise.

For the actual calculation, we use $\frac{1}{m_{u} m_{d}}$ for the $\frac{1}{m_{q} m_{\bar{q}}}$ terms of $\overline{\rho^{ \pm}}, \rho^{0}$ and $\omega^{0}$. For the $\phi$ meson case, recalling that $\phi$ is constituted as $s \bar{s}$, we adopt $\frac{1}{m_{q} m_{\bar{q}}}=\frac{1}{m_{s} m_{s}}$.

Notice that we use the fact that the quark and antiquark masses are equivalent for all cases. This yields the results in Table 2 for the pseudo-scaler case and in Table 3 for the vector meson case.

Here, the estimated mass $M_{\text {est }}$ is obtained by following equation.

$$
M_{\text {est }}=\left(M_{(\text {chiral limit })}^{2}+\left(1+\delta_{S 1}(-1)^{Q+\bar{Q}+1} \alpha_{S} \frac{1}{m_{q} m_{\bar{q}}}\right)\left[\left\langle H_{1}^{\left(\chi_{3}\right)}\right\rangle+\left\langle H_{1}^{\left(\chi_{2}\right)}\right\rangle\right]\right)^{\frac{1}{2}}
$$

where $M_{\text {(chiral limit) }}$ is the meson mass of the chiral limit case, determined by an eigenvalue of non-perturbative Hamiltonian $H_{0}$ in Equation (33) or Equation (34). Recall that these eigenvalues are the same in Ref. [3]. The calculation is performed in the $\mathrm{GeV}$ region. Our corrected light meson mass spectra is better

Table 2. Pseudo-scalar mesons.

\begin{tabular}{|c|c|c|c|c|c|c|}
\hline meson & $f^{0}$ & $\kappa^{+}\left(\kappa^{-}\right)$ & $\kappa^{0}\left(\overline{\kappa^{0}}\right)$ & $\eta$ & $\eta^{\prime}$ & $a_{0}$ \\
\hline quark & $\begin{array}{l}u \bar{u} \cos \theta \\
+d \bar{d} \sin \theta\end{array}$ & $u \bar{s}(\overline{u s})$ & $d \bar{s}(\bar{d} s)$ & $\begin{array}{l}(u \bar{u}+d \bar{d}) \cos \theta \\
-s \bar{s} \sin \theta\end{array}$ & $\begin{array}{l}(u \bar{u}+d \bar{d}) \cos \theta \\
+s \bar{s} \sin \theta\end{array}$ & $\begin{array}{l}u \bar{u} \cos \theta \\
-d \bar{d} \sin \theta\end{array}$ \\
\hline$\kappa^{\gamma_{3}}$ & 1 & 1 & 1 & 2 & 5 & 6 \\
\hline$\kappa^{x_{2}}$ & $\frac{1}{2}$ & $\frac{1}{2}$ & $\frac{1}{2}$ & $\frac{3}{2}$ & $\frac{9}{2}$ & $\frac{11}{2}$ \\
\hline$n$ & 0 & 0 & 0 & 1 & 4 & 5 \\
\hline$\frac{H_{1}^{\left(\chi_{3}\right)}}{\left(m_{1}+m_{2}\right)^{2}}$ & 9.70645 & 9.70645 & 9.70645 & 7.517288 & 7.098759 & 0.009287 \\
\hline$\frac{H_{1}^{\left(\chi_{2}\right)}}{\left(m_{1}+m_{2}\right)^{2}}$ & 2.70136 & 2.70136 & 2.70136 & 8.548402 & 1.790447 & 0.034424 \\
\hline $\cos \theta$ & $\frac{1}{\sqrt{2}}$ & - & - & 0.977172 & 0.503492 & $\frac{1}{\sqrt{2}}$ \\
\hline $\sin \theta$ & $\frac{1}{\sqrt{2}}$ & - & - & 0.21245 & 0.864 & $\frac{1}{\sqrt{2}}$ \\
\hline $\begin{array}{c}M_{e} \\
(\mathrm{MeV})\end{array}$ & 441.339 & 493.672 & 497.623 & 547.816 & 957.772 & 989.780 \\
\hline $\begin{array}{c}M_{m} \\
(\mathrm{MeV})[21]\end{array}$ & $400-550$ & $\begin{array}{l}493.677 \\
( \pm 0.016)\end{array}$ & $\begin{array}{l}497.614 \\
( \pm 0.024)\end{array}$ & $\begin{array}{l}547.862 \\
( \pm 0.018)\end{array}$ & $\begin{array}{l}957.78 \\
( \pm 0.06)\end{array}$ & $\begin{array}{c}980 \\
( \pm 20)\end{array}$ \\
\hline
\end{tabular}

Note: $M_{e}$ and $M_{m}$ denote estimated mass and measured mass, respectively. 
Table 3. Vector mesons.

\begin{tabular}{|c|c|c|c|c|}
\hline meson & $\rho^{ \pm}$ & $\rho^{0}$ & $\omega^{0}$ & $\phi$ \\
\hline quark & $u \bar{d}(\bar{u} d)$ & $u \bar{u} \cos \theta-d \bar{d} \sin \theta$ & $u \bar{u} \cos \theta+d \bar{d} \sin \theta$ & $s \bar{s}$ \\
\hline$\kappa^{\gamma_{3}}$ & 3 & 3 & 4 & 7 \\
\hline & 5 & 5 & 7 & 13 \\
\hline$\kappa^{12}$ & $\overline{2}$ & $\overline{2}$ & $\overline{2}$ & $\overline{2}$ \\
\hline$n$ & 2 & 2 & 3 & 6 \\
\hline$\frac{H_{1}^{\left(\chi_{3}\right)}}{\left(m_{1}+m_{2}\right)^{2}}$ & 0.794996 & 0.794996 & 1.647083 & 0.03221 \\
\hline$\frac{H_{1}^{\left(\chi_{2}\right)}}{\left(m_{1}+m_{2}\right)^{2}}$ & 0.541695 & 0.541695 & 0.017138 & 2.831617 \\
\hline $\cos \theta$ & - & 0.5599 & 0.977 & - \\
\hline $\sin \theta$ & - & 0.82856 & 0.213239 & - \\
\hline$\alpha_{s}$ & 0.0125 & 0.0125 & 0.0125 & 0.0125 \\
\hline $\begin{array}{c}M_{e} \\
(\mathrm{MeV})\end{array}$ & 775.038 & 775.309 & 782.688 & $1018 . .426$ \\
\hline $\begin{array}{c}M_{m} \\
(\mathrm{MeV})[21]\end{array}$ & $775.11 \pm 0.34$ & $775.26 \pm 0.25$ & $782.65 \pm 0.12$ & $1019.461 \pm 0.019$ \\
\hline
\end{tabular}

Note: $\alpha_{s}$ denotes spin parameter.

fit compared to those in Ref. [1] and Ref. [2].

\section{Conclusion}

We obtain plausible light meson mass spectra by invoking the masses of $\mathrm{u}, \mathrm{d}$, and $s$ quarks and the contribution of a spin effect. There is a discrepancy between our values and those of the Particle data group values. However, the Particle data group uses a Lattice QCD approach that shows a Reggae-like function (for examples, see Ref. [7]). As mentioned in the Introduction, the Jefferson Lab Hall A collaboration showed that a Gaussian function is better for fitting to experimental data. Because our estimation is based on a Gaussian-like wave function, our values are still meaningful despite this discrepancy. We consider nonzero quark masses and a spin effect as perturbative corrections of the chiral limit mass spectra given in Ref. [3]. By invoking the masses of $\mathrm{u}, \mathrm{d}$, and s quarks, we can obtain the mass difference between charged kaons $\kappa^{+}\left(\kappa^{-}\right)$and neutral kaons $\kappa^{0}\left(\overline{\kappa^{0}}\right)$ as well as the mass difference between charged $\rho$ mesons $\rho^{ \pm}$ and neutral $\rho$ mesons $\rho^{0}$. The significant point is that the pion mass is unchanged even though quarks become massive. The corresponding pion wave functions are unchanged for $\chi_{3}, \chi_{2}$ and $\chi_{1}$, but $\chi_{0}$ is no longer zero because it is expressed as $\frac{1}{r^{2}} \mathrm{e}^{-\frac{g^{2} L_{1}}{8} r^{2}}$. We then notice the following interesting correspondence to the results from lattice QCD approach. Broniowski et al. [22] showed in NJL model that the pseudo-scaler wave function of pions corresponding 
to $\chi_{0}$ becomes $\frac{1}{r^{\frac{3}{2}}} \mathrm{e}^{-k r}$ and the axial vector wave functions corresponding to $\chi_{3}$ becomes $\frac{1}{r^{\frac{1}{2}}} \mathrm{e}^{-k r}$ when $r$ is large. Because $\chi_{3} \propto \frac{1}{r} \mathrm{e}^{-\frac{g^{2} L_{1}}{8} r^{2}}$, the correspondence that $\frac{1}{r^{2}} \Leftrightarrow \frac{1}{r^{\frac{3}{2}}}$ and $\frac{1}{r} \Leftrightarrow \frac{1}{r^{\frac{1}{2}}}$ are obvious. Because the characteristic part of a pion wave function is Gaussian as shown in Ref. [11] (Gaussian in $r$ space is Gaussian in momentum space), Our pion wave function is very plausible. We next argue for the value of our proposition stated in the Introduction that the first priority governing meson evaluation should be the use of a gauge invariance system instead of a covariance system.

\section{Discussion}

We estimate the masses of $\eta$ and $\eta^{\prime}$ and those of $\rho$ and $\omega$ independently. Usually, the assumption of a mixed state is used to obtain those masses, as in Ref. [2]. Instead, we estimate the masses from different chiral limit masses and calculate each of them within a closed process. However, from the view point of quark contents, $\eta$ and $\eta^{\prime}$ and also $\rho^{0}$ and $\omega^{0}$ are not linearly independent. Precisely, as quark contents, $\eta$ and $\eta^{\prime}$ are usually described as $\eta=\frac{u \bar{u}+d \bar{d}-2 s \bar{s}}{\sqrt{6}}$ and $\eta^{\prime}=\frac{u \bar{u}+d \bar{d}+s \bar{s}}{\sqrt{3}}$ and also $\rho^{0}=\frac{u \bar{u}-d \bar{d}}{\sqrt{2}}$ and $\omega^{0}=\frac{u \bar{u}+d \bar{d}}{\sqrt{2}}$. In our description, $\eta=(u \bar{u}+d \bar{d}) \cos \theta_{\eta}-s \bar{s} \sin \theta_{\eta}$ and $\eta^{\prime}=(u \bar{u}+d \bar{d}) \cos \theta_{\eta^{\prime}}+s \bar{s} \sin \theta_{\eta^{\prime}}$ whereas $\rho^{0}=u \bar{u} \cos \theta_{\rho}-d \bar{d} \sin \theta_{\rho}$ and $\omega^{0}=u \bar{u} \cos \theta_{\omega}+d \bar{d} \sin \theta_{\omega}$. The $\theta$ values in Table 2 and Table 3 show that $\eta$ and $\eta^{\prime}$ and also $\rho^{0}$ and $\omega^{0}$ are not linearly independent. At this time, we cannot interpret the meaning of these results. Also, as previously mentioned in Results, the mass of a pion is unchanged so we cannot obtain the mass difference between $\pi^{ \pm}$and $\pi^{0}$. We consider that the investigation of mass difference between $\pi^{ \pm}$and $\pi^{0}$ might address the question of why a zero mass meson is still unobserved. In other words, we consider that this might clarify whether a pion is a Goldstone boson or not. We can at least say, from our previous results in Ref. [3] and this paper, that a pion is unique among mesons because it has a singularity in its wave function and its mass is unchanged even though its constituent quarks become massive.

\section{Conflicts of Interest}

The author declares no conflicts of interest regarding the publication of this paper.

\section{References}

[1] Braw, F. and Semay, C. (1998) Physical Review D, 58, Article ID: 03015. 
https://doi.org/10.1103/PhysRevD.58.034015

[2] Choi, H.-M., Ji, C.-R., Li, Z. and Ryu, H.-Y. (2015) Physical Review C, 92, Article ID: 055203.

[3] Kurai, T. (2018) Results in Physics, 10, 865. https://doi.org/10.1016/j.rinp.2018.07.034

[4] t'Hooft, G. (1974) Nuclear Physics B, 75, 461. https://doi.org/10.1016/0550-3213(74)90088-1

[5] Hornbostul, K., Brodsky, S. and Pauli, C. (1990) Physical Review D, 41, 3814. https://doi.org/10.1103/PhysRevD.41.3814

[6] Kurai, T. (2014) Progress of Theoretical and Experimental Physics, 2014, 053B01.

[7] Mello, C.S., de Mollo, J.P.B.C. and Frederico, T. (2017) Physics Letters B, 766, 86. https://doi.org/10.1016/j.physletb.2016.12.058

[8] Biernat, E.P., Gross, F., Pena, M.T. and Stadler, A. (2014) Physical Review D, 89, Article ID: 016006. https://doi.org/10.1103/PhysRevD.89.016005

[9] Chang, L., Cloet, I.C., Roberts, C.D., Schmidt, S.M. and Tandy, P.C. (2013) Physical Review Letters, 111, Article ID: 141802. https://doi.org/10.1103/PhysRevLett.111.141802

[10] Arrington, J., et al. (2021) Revealing the Structure of Light Pseudoscalar Mesons at the Electron-Ion Collider.

[11] Li, Y., Maris, P. and Vary, J.P. (2018) Physical Review D, 97, Article ID: 054034. https://doi.org/10.1103/PhysRevD.97.054034

[12] Dlamini, et al. (2020) Deep Exclusive Electroproduction of $\pi^{\wedge} 0$ at High $\mathrm{Q}^{\wedge} 2$ in the Quark Valence Regime.

[13] Aoki, K., Bando, M., Kugo, T. and Nakanishi, H. (1990) Progress of Theoretical Physics, 84, 683. https://doi.org/10.1143/ptp/84.4.683

[14] Weinberg, S. (2000) The Quantum Theory of Fields III. Press Syndicate University, Cambridge. https://doi.org/10.1017/CBO9781139644198

[15] Suura, H. (1978) Physical Review D, 17, 469. https://doi.org/10.1103/PhysRevD.17.469

[16] Suura, H. (1979) Physical Review D, 20, 1412. https://doi.org/10.1103/PhysRevD.20.1412

[17] Kurai, T. (2017) Results in Physics, 7, 2066. https://doi.org/10.1016/j.rinp.2017.05.028

[18] Moriguchi, S., Udagawa, K. and Hitotsumatsu, S. (1975) Formula of Mathematics 3 Special Functions, Iwanami.

[19] Gradshteyn, I.S. and Ryzhik, M. (1980) Table of Integrals, Series and Products. Academic Press, Cambridge.

[20] Zyla, P.A., et al. (2020) Progress of Theoretical and Experimental Physics, 2020, $083 \mathrm{C} 01$.

[21] Olive, K.A., et al. (2014) Chinese Physics C, 38, Article ID: 090001. https://doi.org/10.1088/1674-1137/38/9/090001

[22] Broniowski, W., Prelovsec, S., Santeij, I. and Arriora, E.R. (2010) Physics Letters B, 686,313 


\section{Appendix A. Derivation of Kinetic Terms}

In Equation (9) we obtain the following.

$$
\text { Kinetic term }=-[i \vec{\alpha} \cdot \vec{\nabla}(r), q(r)]
$$

Here, we deal only with space coordinates so that the t variable is omitted.

In order to evaluate Equation (A1), we use the fact that

$$
\vec{\alpha} \cdot \vec{\nabla}(r)=\alpha^{1} \frac{\partial}{\partial x_{1}}+\alpha^{2} \frac{\partial}{\partial x_{2}}+\alpha^{3} \frac{\partial}{\partial x_{3}}
$$

and the decomposition of $q(r)$ given Equation (12). For the $\beta$ and $\beta(i \vec{\alpha} \cdot \hat{r})$ components, the derivation is given in Appendix D of Ref. [17]. Thus, we can derive the $(-i \vec{\alpha} \cdot \hat{r})$ component and Unit matrix component.

For $(-i \vec{\alpha} \cdot \hat{r})$ component, we obtain the following

$$
\vec{\alpha} \cdot \vec{\nabla}(r)=\vec{\alpha} \cdot \hat{r} \frac{\partial}{\partial r}
$$

The considered commutation equation becomes as follows.

$$
-\left[i \vec{\alpha} \cdot \hat{r} \frac{\partial}{\partial r}, 1 q_{0}(r)\right]=(-i \vec{\alpha} \cdot \hat{r})\left[\frac{\partial}{\partial r}, q_{0}(r)\right]=0
$$

For Unit matrix component, we use Equation (A2). The commutation equation becomes the following

$$
\begin{aligned}
- & {\left[i \vec{\alpha} \cdot \vec{\nabla}(r),(-i \vec{\alpha} \cdot \hat{r}) q_{1}(r)\right] } \\
= & -\left(\alpha^{1} \frac{\partial}{\partial x_{1}}+\alpha^{2} \frac{\partial}{\partial x_{2}}+\alpha^{3} \frac{\partial}{\partial x_{3}}\right) \times \frac{\alpha^{1} x_{1}+\alpha^{2} x_{2}+\alpha^{3} x_{3}}{r} q_{1}(r) \\
& +\frac{\alpha^{1} x_{1}+\alpha^{2} x_{2}+\alpha^{3} x_{3}}{r} \times\left(\alpha^{1} \frac{\partial}{\partial x_{1}}+\alpha^{2} \frac{\partial}{\partial x_{2}}+\alpha^{3} \frac{\partial}{\partial x_{3}}\right) q_{1}(r)
\end{aligned}
$$

The first term of Equation (A4) is written as below.

$$
\begin{aligned}
\text { First term }= & -\left(\alpha^{1} \frac{\partial}{\partial x_{1}}(\vec{\alpha} \cdot \hat{r})+\alpha^{2} \frac{\partial}{\partial x_{2}}(\vec{\alpha} \cdot \hat{r})+\alpha^{3} \frac{\partial}{\partial x_{3}}(\vec{\alpha} \cdot \hat{r})\right) q_{1}(r) \\
& -\alpha^{1}(\vec{\alpha} \cdot \hat{r}) \frac{\partial}{\partial x_{1}} q_{1}(r)-\alpha^{2}(\vec{\alpha} \cdot \hat{r}) \frac{\partial}{\partial x_{2}} q_{1}(r)-\alpha^{3}(\vec{\alpha} \cdot \hat{r}) \frac{\partial}{\partial x_{3}} q_{1}(r)
\end{aligned}
$$

In order to evaluate the upper part of Equation (A5) further, we use the following result.

For $l \neq m$ case

$$
\begin{aligned}
\alpha^{l} \frac{\partial}{\partial x_{l}} \frac{\alpha^{m} x_{m}}{r} & =\frac{1}{2}\left(\alpha^{l} \frac{\partial}{\partial x_{l}} \frac{\alpha^{m} x_{m}}{r}+\alpha^{m} \frac{\partial}{\partial x_{m}} \frac{\alpha^{l} x_{l}}{r}\right) \\
& =\frac{1}{2}\left(\alpha^{l} \alpha^{m} \frac{-x_{m} x_{l}}{r^{3}}+\alpha^{m} \alpha^{l} \frac{-x_{l} x_{m}}{r^{3}}\right) \\
& =-\frac{1}{2}\left[\alpha^{l}, \alpha^{m}\right]_{+} \frac{x_{l} x_{m}}{r^{3}} \\
& =-\frac{1}{2}\left[\gamma^{l}, \gamma^{m}\right]_{+} \frac{x_{l} x_{m}}{r^{3}} \\
& =-\eta^{l m} \frac{x_{l} x_{m}}{r^{3}}=0
\end{aligned}
$$


Here we use the fact that $\gamma^{l} \gamma^{m}+\gamma^{m} \gamma^{l}=2 \eta^{l m}, \eta^{l m}=1(l=m)$ and $\eta^{l m}=0(l \neq m)$.

For $l=m$ case, we obtain the following.

$$
-\left(\alpha^{1} \frac{\partial}{\partial x_{1}} \frac{\alpha^{1} x_{1}}{r}+\alpha^{2} \frac{\partial}{\partial x_{2}} \frac{\alpha^{2} x_{2}}{r}+\alpha^{3} \frac{\partial}{\partial x_{3}} \frac{\alpha^{3} x_{3}}{r}\right)=-\left(\frac{3}{r}-\frac{x_{1}^{2}+x_{2}^{2}+x_{3}^{2}}{r^{3}}\right)=-\frac{2}{r}
$$

Together with the second term of Equation (A4), the lower term of Equation (A5) becomes as below.

$$
\begin{aligned}
& -\alpha^{l} \frac{\alpha^{m} x_{m}}{r} \frac{\partial}{\partial x_{l}} q_{1}(r)+\frac{\alpha^{m} x_{m}}{r} \alpha^{l} \frac{\partial}{\partial x_{l}} q_{1}(r) \\
& =-\alpha^{l} \frac{\alpha^{m} x_{m}}{r} \frac{\partial}{\partial x_{l}} q_{1}(r)+\frac{\alpha^{l} x_{l}}{l} \alpha^{m} \frac{\partial}{\partial x_{m}} q_{1}(r) \\
& =-\alpha^{l} \alpha^{m} \frac{1}{r}\left(x_{m} \frac{\partial}{\partial x_{l}}-x_{l} \frac{\partial}{\partial x_{m}}\right) q_{1}(r)
\end{aligned}
$$

For the second term in the first line, we exchange $l$ and $m$ because $\alpha^{l} x_{l}\left(\alpha^{m} x_{m}\right)$ denotes a summation convention. We use the following polar coordinate description.

$$
\begin{gathered}
x_{1}=r \sin \theta \cos \phi, x_{2}=r \sin \theta \sin \phi, x_{3}=r \cos \theta \\
\frac{\partial}{\partial x_{1}}=\sin \theta \cos \phi \frac{\partial}{\partial r}+\frac{\cos \theta \cos \phi}{r} \frac{\partial}{\partial \theta}-\frac{\sin \phi}{r \sin \theta} \frac{\partial}{\partial \phi} \\
\frac{\partial}{\partial x_{2}}=\sin \theta \sin \phi \frac{\partial}{\partial r}+\frac{\cos \theta \sin \phi}{r} \frac{\partial}{\partial \theta}+\frac{\cos \phi}{r \sin \theta} \frac{\partial}{\partial \phi} \\
\frac{\partial}{\partial x_{3}}=\cos \theta \frac{\partial}{\partial r}-\frac{\sin \theta}{r} \frac{\partial}{\partial \theta}
\end{gathered}
$$

Because $q_{1}$ is only the function of $r, \frac{\partial q_{1}}{\partial \theta}=0$ and $\frac{\partial q_{1}}{\partial \phi}=0$.

Then,

$$
\left(x_{m} \frac{\partial}{\partial x_{l}}-x_{l} \frac{\partial}{\partial x_{m}}\right) q_{1}(r)=0
$$

Thus, reviving the time variable and sandwiching $q_{1}(t ; r)$ with the vacuum state and the physical state, Unit matrix component of kinetic term becomes the following.

Unit matrix component: $-\frac{2}{r} \chi_{1}(r)$

Appendix B. Evaluation of $\int_{0}^{\infty} \mathrm{d} z \exp \left(-\frac{z^{2}}{2}\right) \frac{\sinh (\gamma z)}{z}$

To evaluate the integral, we use the following formula [19].

$$
\begin{aligned}
& \int_{0}^{\infty} \mathrm{d} x x^{2 \mu-1} \mathrm{e}^{-\beta x^{2}} \sinh (\gamma x) \\
& =\frac{1}{2} \Gamma(2 \mu)(2 \beta)^{-\mu} \exp \left(\frac{\gamma^{2}}{8 \beta}\right)\left[D_{-2 \mu}\left(-\frac{\gamma}{\sqrt{2 \beta}}\right)-D_{-2 \mu}\left(\frac{\gamma}{\sqrt{2 \beta}}\right)\right]
\end{aligned}
$$


$D_{\lambda}(z)$ is the following Weber function, as defined in Ref. [18].

$$
D_{\lambda}(z)=2^{\frac{\lambda}{2}} \sqrt{\pi} \mathrm{e}^{-\frac{z^{2}}{4}}\left[\frac{1}{\Gamma\left(\frac{1-\lambda}{2}\right)} F\left(-\frac{\lambda}{2}, \frac{1}{2} ; \frac{z^{2}}{2}\right)-\frac{\sqrt{2} z}{\Gamma\left(-\frac{\lambda}{2}\right)} F\left(\frac{1-\lambda}{2}, \frac{3}{2} ; \frac{z^{2}}{2}\right)\right]
$$

where $F(\alpha, \gamma ; z)$ is confluent hyper geometric series, as shown in Equation (40).

Because, $\beta$ is $1 / 2$ for our case, the Weber function term simplifies to $D_{\lambda}(-\gamma)-D_{\lambda}(\gamma)$.

Here we replace $-2 \mu$ with $\lambda$. To calculate this term, we use the following recursion formula from Ref. [18].

$$
D_{\lambda}(z)=\frac{\Gamma(\lambda+1)}{\sqrt{2 \pi}}\left[i^{\lambda} D_{-\lambda-1}(i z)+(-i)^{\lambda} D_{-\lambda-1}(-i z)\right]
$$

The difference of the Weber function term becomes as follows.

$$
\begin{aligned}
& D_{-\lambda}(-z)-D_{-\lambda}(z) \\
& =\frac{\Gamma(-\lambda+1)}{\sqrt{2 \pi}} i\left[\left(i^{-\lambda}-(-i)^{-\lambda}\right) D_{\lambda-1}(-i z)+\left((-i)^{-\lambda}-i^{-\lambda}\right) D_{\lambda-1}(i z)\right]
\end{aligned}
$$

Because $i^{-\lambda}=\mathrm{e}^{i \frac{\pi}{2}(-\lambda)}=\mathrm{e}^{-i \frac{\pi}{2} \lambda}$, Equation (A9) is expressed as below.

$$
\begin{aligned}
& D_{-\lambda}(-z)-D_{-\lambda}(z) \\
& =\frac{\Gamma(-\lambda+1)}{\sqrt{2 \pi}}\left[\left(\mathrm{e}^{-i \frac{\pi}{2} \lambda}-\mathrm{e}^{i \frac{\pi}{2} \lambda}\right) D_{\lambda-1}(-i z)+\left(\mathrm{e}^{i \frac{\pi}{2} \lambda}-\mathrm{e}^{-i \frac{\pi}{2} \lambda}\right) D_{\lambda-1}(i z)\right] \\
& =\frac{\Gamma(-\lambda+1)}{\sqrt{2 \pi}} 2 i \sin \left(\frac{\pi}{2} \lambda\right)\left[-D_{\lambda-1}(-i z)+D_{\lambda-1}(i z)\right]
\end{aligned}
$$

Here, using the definition of a Weber function as shown in Equation (A6), we obtain the following.

$$
-D_{\lambda-1}(-i z)+D_{\lambda-1}(i z)=2^{\frac{\lambda-1}{2}} \sqrt{\pi} \mathrm{e}^{\frac{z^{2}}{4}}(-1) \frac{2 \sqrt{2} i z}{\Gamma\left(\frac{1-\lambda}{2}\right)} F\left(\frac{2-\lambda}{2}, \frac{3}{2} ;-\frac{z^{2}}{2}\right)
$$

Thus,

$$
\begin{aligned}
& D_{-\lambda}(-z)-D_{-\lambda}(z) \\
& =\frac{\Gamma(-\lambda+1)}{\sqrt{2 \pi}}(2 i) \sin \left(\frac{\pi}{2} \lambda\right) 2^{\frac{\lambda-1}{2}} \sqrt{\pi} \mathrm{e}^{\frac{z^{2}}{4}} \frac{2 \sqrt{2}(-i z)}{\Gamma\left(\frac{1-\lambda}{2}\right)} F\left(\frac{2-\lambda}{2}, \frac{3}{2} ;-\frac{z^{2}}{2}\right) \\
& =\Gamma(-\lambda+1) 4 \sin \left(\frac{\pi}{2} \lambda\right) 2^{\frac{\lambda-1}{2}} \mathrm{e}^{\frac{z^{2}}{4}} \frac{z}{\Gamma\left(\frac{1-\lambda}{2}\right)} F\left(\frac{2-\lambda}{2}, \frac{3}{2} ;-\frac{z^{2}}{2}\right)
\end{aligned}
$$

Taking $\lambda=2 \varepsilon$ and allowing $\varepsilon$ to approach 0, Equation (A6) becomes the following. 


$$
\begin{aligned}
& \int_{0}^{\infty} \mathrm{d} x \mathrm{e}^{-\frac{x^{2}}{2}} \frac{\sinh (\gamma x)}{x}=\lim _{\varepsilon \rightarrow 0} \frac{1}{2} \Gamma(2 \varepsilon)(-1)^{-\varepsilon} \mathrm{e}^{\frac{\gamma^{2}}{4}} \Gamma(-2 \varepsilon+1) 4 \sin (\varepsilon \pi) 2^{\frac{2 \varepsilon-1}{2}} \mathrm{e}^{\frac{\gamma^{2}}{4}} \\
& \times \frac{\gamma}{\Gamma\left(\frac{1-2 \varepsilon}{2}\right)} F\left(1-\varepsilon, \frac{3}{2} ;-\frac{\gamma^{2}}{2}\right) \\
& =\lim _{\varepsilon \rightarrow 0} \frac{1}{2} \frac{1}{2 \varepsilon} \mathrm{e}^{\frac{\gamma^{2}}{2}} \Gamma(1) 4(\varepsilon \pi) \frac{1}{\sqrt{2}} \frac{\gamma}{\Gamma\left(\frac{1}{2}\right)} F\left(1, \frac{3}{2} ;-\frac{z^{2}}{2}\right) \\
& =\sqrt{\frac{\pi}{2}} \mathrm{e}^{\frac{\gamma^{2}}{2}} \gamma F\left(1, \frac{3}{2} ;-\frac{\gamma^{2}}{2}\right)
\end{aligned}
$$

where $\gamma=\frac{\overline{P_{0}^{\prime}}}{\sqrt{\frac{g^{2} L_{1}}{2}}}$.

For the last line, we use the fact that $\Gamma(1)=1, \Gamma\left(\frac{1}{2}\right)=\sqrt{\pi}$.

Note that Equation (67) is obtained by multiplying this form by a factor 2 .

To obtain an approximation form of Equation (67), we use the following integral representation form of a confluent hypergeometric series [19].

$$
F(\alpha, \zeta ; z)=\frac{1}{B(\alpha, \zeta-\alpha)} z^{1-\zeta} \int_{0}^{z} \mathrm{~d} t \mathrm{e}^{t} t^{\alpha-1}(z-t)^{\zeta-\alpha-1}
$$

where $B(\alpha, \zeta-\alpha)$ is the Beta function below, as defined in Ref. [18].

$$
B(p, q)=\int_{0}^{1} \mathrm{~d} t t^{p-1}(1-t)^{q-1}
$$

Because $\alpha=1, \zeta=\frac{3}{2}, z=-\frac{\gamma^{2}}{2}$ in our case, Equation (A13) becomes as below.

$$
\begin{aligned}
F\left(1, \frac{3}{2} ;-\frac{\gamma^{2}}{2}\right) & =\frac{1}{B\left(1, \frac{1}{2}\right)}\left(-\frac{\gamma^{2}}{2}\right)^{-\frac{1}{2}} \int_{0}^{-\frac{\gamma^{2}}{2}} \mathrm{~d} t \mathrm{e}^{t}\left(-\frac{\gamma^{2}}{2}-t\right)^{-\frac{1}{2}} \\
& =\frac{1}{B\left(1, \frac{1}{2}\right)} \frac{1}{\left(\frac{\gamma^{2}}{2}\right)^{\frac{1}{2}}} \int_{-\frac{\gamma^{2}}{2}}^{0} \mathrm{~d} t \mathrm{e}^{t}\left(t+\frac{\gamma^{2}}{2}\right)^{-\frac{1}{2}}
\end{aligned}
$$

With the variable substitution $t=-u$, the integral part of Equation (A15) becomes the following.

$$
I=\int_{0}^{\frac{\gamma^{2}}{2}} \mathrm{~d} u \frac{\mathrm{e}^{-u}}{\sqrt{\frac{\gamma^{2}}{2}-u}} \approx \mathrm{e}^{-\frac{\gamma^{2}}{2}}
$$

The last result is obtained by the consideration that the maximum contribution to this integral comes from $u=\frac{\gamma^{2}}{2}$. 
Because, using Equation (A13) and Equation (A14), $B\left(1, \frac{1}{2}\right)$ is easily calculated as $B\left(1, \frac{1}{2}\right)=2$.

Equation (A15) becomes as follows.

$$
F\left(1, \frac{3}{2} ;-\frac{\gamma^{2}}{2}\right)=\frac{1}{\sqrt{2} \gamma} \mathrm{e}^{-\frac{\gamma^{2}}{2}}
$$

Then, final result is obtained as Equation $(\mathrm{A} 12) \approx \frac{\sqrt{\pi}}{2}$.

Thus, after multiplying by a factor 2, the approximated form of Equation (68) becomes the following.

$$
\text { Eq. }(68) \approx \sqrt{\pi} \exp \left(-\frac{\overline{P_{0}^{\prime 2}}}{g^{2} L_{1}}\right)
$$

This is the form of Equation (69).

\section{Appendix C. Evaluation of Mass of $\kappa^{ \pm}$and $\kappa^{0}$}

The nonperturbative wave function of a kaon is expressed as follows:

$$
\begin{aligned}
& \chi_{3}^{(\kappa)}(r)=r \exp \left(-\frac{g^{2} L_{1}}{8} r^{2}\right) \quad\left(\kappa^{\chi_{3}}=1\right. \text { for Equation (39)) } \\
& \chi_{2}^{(\kappa)}=\exp \left(-\frac{g^{2} L_{1}}{8} r^{2}\right) \quad\left(\kappa^{\chi_{2}}=\frac{1}{2}\right. \text { for Equation (43)) }
\end{aligned}
$$

We show here, as an example, how to evaluate numerator of $\left\langle H_{1}^{\left(\chi_{3}\right)}\right\rangle$, the integral part of Equation (61) becomes as below.

$$
I_{1}^{\left(\chi_{3}\right)}=\int_{-\infty}^{\infty} \mathrm{d} z \exp \left(-\frac{1}{2}(z-\gamma)^{2}\right) \frac{(z-\gamma)^{4}}{z}
$$

where $\gamma=\overline{P_{0}^{\prime}} / \sqrt{\frac{g^{2} L_{1}}{2}}$, The factor $4 \pi$ is omitted because is cancelled out by denominator calculation.

$$
I_{1}^{\left(\chi_{3}\right)}=\int_{-\infty}^{0} \mathrm{~d} z \exp \left(-\frac{1}{2}(z-\gamma)^{2}\right) \frac{(z-\gamma)^{4}}{z}+\int_{0}^{\infty} \mathrm{d} z \exp \left(-\frac{1}{2}(z-\gamma)^{4}\right) \frac{(z-\gamma)^{2}}{z}
$$

For the first term, use of a variable substitution $z=-z^{\prime}$ converts Equation (A16) to the following.

$$
\begin{aligned}
I_{1}^{\left(\chi_{3}\right)}= & \mathrm{e}^{-\frac{\gamma^{2}}{2}}(-1) \int_{0}^{\infty} \mathrm{d} z \mathrm{e}^{-\frac{z^{2}}{2}} \mathrm{e}^{-\gamma z}\left(z^{3}+4 z^{2} \gamma+6 z \gamma^{2}+4 \gamma^{3}+\frac{\gamma^{4}}{z}\right) \\
& +\mathrm{e}^{-\frac{\gamma^{2}}{2}} \int_{0}^{\infty} \mathrm{d} z \mathrm{e}^{-\frac{z^{2}}{2}} \mathrm{e}^{\gamma z}\left(z^{3}-4 z^{2} \gamma+6 z \gamma^{2}-4 \gamma^{3}+\frac{\gamma^{4}}{z}\right) \\
= & \mathrm{e}^{-\frac{\gamma^{2}}{2}} \int_{0}^{\infty} \mathrm{d} z \mathrm{e}^{-\frac{z^{2}}{2}}\left[z^{3}\left(\mathrm{e}^{\gamma z}-\mathrm{e}^{-\gamma z}\right)-4 z^{2}\left(\mathrm{e}^{\gamma z}+\mathrm{e}^{-\gamma z}\right) \gamma\right. \\
& \left.+6 z\left(\mathrm{e}^{\gamma z}-\mathrm{e}^{-\gamma z}\right) \gamma^{2}-4\left(\mathrm{e}^{\gamma z}+\mathrm{e}^{-\gamma z}\right) \gamma^{3}+\frac{\mathrm{e}^{\gamma z}-\mathrm{e}^{-\gamma z}}{z} \gamma^{4}\right]
\end{aligned}
$$




$$
\begin{aligned}
= & \mathrm{e}^{-\frac{\gamma^{2}}{2}} 2\left(\int_{0}^{\infty} \mathrm{d} z \mathrm{e}^{-\frac{z^{2}}{2}} z^{3} \sinh (\gamma z)-4 \int_{0}^{\infty} \mathrm{d} z \mathrm{e}^{-\frac{z^{2}}{2}} z^{2} \cosh (\gamma z) \gamma\right. \\
& +6 \int_{0}^{\infty} \mathrm{d} z \mathrm{e}^{-\frac{z^{2}}{2}} z \sinh (\gamma z) \gamma^{2}-4 \int_{0}^{\infty} \mathrm{d} z \mathrm{e}^{-\frac{z^{2}}{2}} \cosh (\gamma z) \gamma^{3} \\
& \left.+\int_{0}^{\infty} \mathrm{d} z \mathrm{e}^{-\frac{z^{2}}{2}} \frac{\sinh (\gamma z)}{z} \gamma^{4}\right)
\end{aligned}
$$

For the last integral, we use the description of Equation (A11) multiplied by factor 2. For the other integrals, we use the following formula from Ref [19].

$$
\begin{gathered}
\int_{0}^{\infty} \mathrm{d} z \mathrm{e}^{-\beta z^{2}} \cosh (\gamma z)=\frac{1}{2} \sqrt{\frac{\pi}{\beta}} \exp \left(\frac{\gamma^{2}}{4 \beta}\right) \\
\int_{0}^{\infty} \mathrm{d} z \mathrm{e}^{-\beta z^{2}} z \sinh (\gamma z)=\frac{\gamma}{4 \beta} \sqrt{\frac{\pi}{\beta}} \exp \left(\frac{\gamma^{2}}{4 \beta}\right) \\
\int_{0}^{\infty} \mathrm{d} z \mathrm{e}^{-\beta z^{2}} z^{2} \cosh (\gamma z)=\frac{\sqrt{\pi}\left(2 \beta+\gamma^{2}\right)}{8 \beta^{2} \sqrt{\beta}} \exp \left(\frac{\gamma^{2}}{4 \beta}\right)
\end{gathered}
$$

To obtain $z^{3}$ integral, we use integration by parts as below.

$$
\begin{aligned}
& \int_{0}^{\infty} \mathrm{d} z \mathrm{e}^{-\frac{z^{2}}{2}} \sinh (\gamma z) z \\
& =\left[\mathrm{e}^{-\frac{z^{2}}{2}} \sinh (\gamma z) \frac{z^{2}}{2}\right]_{0}^{\infty}-\int_{0}^{\infty} \mathrm{d} z(-z) \mathrm{e}^{-\frac{z^{2}}{2}} \sinh (\gamma z) \frac{z^{2}}{2}-\int_{0}^{\infty} \mathrm{d} z \mathrm{e}^{-\frac{z^{2}}{2}} \gamma \cosh (\gamma z) \frac{z^{2}}{2}
\end{aligned}
$$

Thus,

$$
\int_{0}^{\infty} \mathrm{d} z \mathrm{e}^{-\frac{z^{2}}{2}} z^{3} \sinh (\gamma z)=2 \int_{0}^{\infty} \mathrm{d} z \mathrm{e}^{-\frac{z^{2}}{2}} z \sinh (\gamma z)+\gamma \int_{0}^{\infty} \mathrm{d} z \mathrm{e}^{-\frac{z^{2}}{2}} z^{2} \cosh (\gamma z)
$$

For Equations (A18)-(A20), taking $\beta=\frac{1}{2}$, the first four terms yields

$$
J=\left(-\gamma^{3}+2 \gamma^{2}-2 \gamma\right) \sqrt{2 \pi} \mathrm{e}^{\frac{\gamma^{2}}{2}}
$$

